\title{
Fasti dei sacerdoti del culto di Vulcano ad Ostia
}

\author{
Maria Letizia CALDelLi
}

\section{L. Caldelli, Università di Roma La Sapienza, marialetizia.caldelli@uniromal.it}

Come è noto, il tratto distintivo del panorama religioso ostiense è costituito dal culto di Vulcano. Ciononostante, nella letteratura scientifica è stato trascurato l'importante contributo offerto dai frammenti dei Fasti dei sacerdoti minori del culto, aediles e praetores. Partendo da questi e riprendendo in esame le numerose iscrizioni che li riguardano si è cercato di ricostruire il tessuto sociale che li ha prodotti, individuando nell'età di Commodo un momento di svolta nel reclutamento. Tale svolta può essere interpretata come il riflesso di una maggiore ingerenza di Roma in un culto che, ai suoi massimi livelli, aveva il controllo su tutte le aree sacre della colonia.

Ostia, culto di Vulcano, Fasti, società, Commodo

As we know, the most distinctive feature in the history of Ostia's religion is the pre-eminence of the cult of Vulcan. Nevertheless, in the scientific literature has been overlooked the important contribution offered by the fragments of the Fasti of minor cult priests, aediles and praetores. Starting from these and taking into consideration the numerous inscriptions which concern them we tried to reconstruct the social context that has produced them, identifying the age of Commodus a turning point in recruitment. This turning point can be interpreted as a reflection of greater Rome's interference in a cult that, at its highest levels, had control over the sacred areas of the colony.

Ostia, cult of Vulcan, Fasti, society, Commodus

"The most distinctive feature in the history of Ostia's religion is the pre-eminence of the cult of Vulcan ${ }^{1}$. Con queste parole Russell Meiggs inizia la trattazione dei culti repubblicani nella colonia, dedicando proprio a Vulcano un lungo passaggio. Dei tanti problemi con ciò connessi, non è mia intenzione tornare né sulla questione della introduzione del culto ad Ostia, né su quella della localizzazione del medesimo: su entrambe molto si è scritto, anche di recente ${ }^{2}$. Mi soffermerò invece sui documenti relativi agli attori del culto.

* Desidero ringraziare Giovanni Mennella e Fausto Zevi, lettori attenti e critici del testo che qui si pubblica, la cui responsabilità è ovviamente di chi scrive.

1. Meiggs 19732, p. 337.

2. Sull'introduzione del culto ad Ostia vd. da ultimo Zevi 2009 , p. 503-513, che ripercorre la storia della questione ed avanza una nuova proposta. Sulla localizzazione del culto una sintesi è in Steuernagel 2004, p. 161-165, ma vd. da ultimo Zevi 2012, p. 559-563 (tempio dell'Ara Rotonda).
PONTIFICES VOLCANI

ET AEDIUM SACRARUM

Conosciamo la loro attività da iscrizioni e da numerosi frammenti dei Fasti Ostienses. Rispetto alle analisi di Grosso $^{3}$ e di Meiggs ${ }^{4}$ possiamo aggiungere :

1) la cronologia del primo pontefice noto, P. Lucilius Gamala Senior, va anticipata alla metà del I secolo a.C. (non dunque età augustea) ${ }^{5}$.

3. Grosso 1959 , p. 283-296, part. p. 296 con elenco riassuntivo.

4. Meiggs 19732, p. 514, ma vd. anche p. 514-516.

5. Sulla cronologia di Gamala Senior fanno il punto della situazione numerosi contributi compresi in Gallina ZeviHumphrey 2004. Un decisivo avanzamento negli studi consente oggi di distinguere tra la cronologia del personaggio e l'epoca di redazione del testo. Quanto alla prima, pioniere nel rialzarla al I secolo a.C. è stato Zevi 1973, a cui si rimanda, anche se oggi, rispetto ad allora, lo studioso sembra più incline a scendere alla seconda metà del I a.C., sulla suggestione di D'Arms 2000: vd. dunque Zevi 2004. Ancora all'età augustea pensa Salomies 2003. Quanto alla 
2) M. Naevius Optatus è ora noto, oltreché dai Fasti cittadini, anche da un'iscrizione di recente pubblicazione, sulla quale sarebbe opportuno ritornare ${ }^{6}$.

3) M. Acilius Priscus Egrilius Plarianus, che, come è noto dai Fasti cittadini, entra in carica nel 105, è ancora vivo, dunque pontefice, certamente nel 125/126, quando è praefectus aerari Saturni, e assai probabilmente ancora nel 129 o 130, quando potrebbe aver ricoperto il consolato suffetto. La nuova cronologia del personaggio, dovuta agli studi di Zevi degli anni ' $70^{7}$, permette dunque di escludere il pontificato di C. Suetonius Tranquillus, che, secondo un'iscrizione di Ippona, sarebbe dovuto cadere prima della destituzione nel 121 d.C. ${ }^{8}$ Se questo risolve il problema della presenza ad Ostia di un pontefice, la cui famiglia risulta assente tra le gentes attestate nella colonia, apre la questione della presenza del pontificato di Vulcano in un ulteriore centro del Mediterraneo, altro da Ostia.

4) A. Egrilius Plarianus, probabilmente il figlio dell'omonimo console suffetto del 128 d.C. ${ }^{9}$, tenne il pontificato di Vulcano non prima del 125/126, ma assai probabilmente dopo almeno il 129 o 130, quando suo zio, allora probabilmente console suffetto, era ancora vivente e dunque pontefice in carica.

5) Dubbia rimane l'introduzione nella lista di T. Statilius Taurianus, noto da un'iscrizione del Serapeo, in cui per primo Vidman propose di integrare, in relazione al personaggio, la carica

seconda, alla piena età augustea data per considerazioni interne al testo, ma anche appuntandosi all'impaginazione del medesimo Panciera 2004; al II secolo d.C. pensa invece Lo Cascio 2004, in primis per la terminologia utilizzata nell'indicazione della carica di curator pecuniae publicae exigendae et adtribuendae in comitiis.

6. Marinucci 2012, p. $94-96 \mathrm{n}^{\circ} 118$. Come accenna in nota Marinucci, lo stesso personaggio compare anche in un'iscrizione, solo parzialmente edita, ricomposta da F. Zevi: vd. $C I L$, XIV $4610+4661+4711$ (= Bloch 1958, p. 213 $\left.\mathrm{n}^{\circ} 6\right)+$ inedito inv. 9196.

7. Zevi 1970, p. 289-308, fondamentale per la nuova datazione p. 300-303 nr. 7. Vd. anche Salomies 1996, p. 72.

8. La questione si fonda sull'iscrizione pubblicata da MarecPflaum 1952, p. 76-85, cfr. $A E 1953,73$. Trovata nel foro di Ippona, in essa si integra e si legge alla r. 5: [p]on[t(ifici)] Volca[- - -]. Sull'iscrizione sono tornati di recente Baurain 1976, p. 124-126; Wardle 2002, p. 465-470. Sul cursus di Svetonio vd. PIR2 S 959. Svetonio, inserito sia pur dubitativamente nella lista di Meiggs 19732, p. 514 con considerazioni p. 514-516, viene rimosso negli addenda a p. 584

9. Zevi 1970, p. 302, 309. Vd. anche Salomies 1996, p. 72. di pont(ifex) Vul(kani) et aed(ium) sacr(arum) ${ }^{10}$. Sconsigliano l'introduzione, secondo F. Zevi, non tanto la cronologia del personaggio (ca. 150/160 d.C.), quanto piuttosto l'integrazione della carica, che costringerebbe ad abbreviazioni non consuete, e la non pertinenza dell'intervento pontificale nella concessione di un suolo già proprietà del Caseggiato di Bacco e Arianna (non dunque un suolo relativo ad un'aedes pubblica delle colonia) ${ }^{11}$.

6) È prudente espungere dalla lista di Grosso il nome di Granius Roma[nus?], che compare in un'iscrizione di Porto, senza indicazione della carica rivestita a causa di una profonda lacuna ${ }^{12}$. L'accostamento tra il greco epì + il genitivo del nome con l'espressione permissu seguita da un nome e dalla carica di pontifex Volcani che compare in un'altra iscrizione bilingue di Porto, non è sembrata e non sembra argomento sufficiente ${ }^{13}$.

7) Alla lista dei pontefici noti occorre aggiungere Q. Vettius Postumius Constantius, che tenne la carica sullo scorcio del III secolo ${ }^{14}$.

\section{AEDILES E PRAETORES VOLCANI}

Definiti nella letteratura come «assistenti nel culto ${ }^{15}$, non sappiamo quali mansioni esattamente avessero. Se un passaggio dei Fasti relativo all'anno 91 d.C. si riferisce a loro, ne dobbiamo concludere che avessero almeno alcune competenze nel neutralizzare gli oggetti colpiti da un fulmine ${ }^{16}$.

10. Vidman 1969, n 533c. Lo segue Pellegrino 1988, p. 239, cfr. $A E 1988,216$.

11. Zevi 2004, p. 107-108.

12. $I G, \mathrm{XIV} 914$.

13. Grosso 1959, p. 294-295 (a. 222-226). Contra Meiggs 19732, p. 514; IGI. Porto, $\mathrm{n}^{\circ} 17$, p. 41.

14. CIL, XIV $132+$ fr. ined. inv. $6418=$ Balbi de Caro 1968 , p. $75-82$, cfr. $A E 1968,81$ : pur proponendo la data del 287 d.C., che vedrebbe insieme consoli Diocleziano per la terza volta e Massimiano per la prima, non esclude altre date, quali il 290, 293, 299, 303 e 304, che pure videro insieme come consoli Diocleziano e Massimiano. Meiggs 19732, p. 514 conosceva ovviamente $C I L$, XIV 132, che, in considerazione del suo stato frammentario, non gli permetteva di ricavare il nome del pontefice di Vulcano e, d'altra parte, imponeva una datazione non anteriore al IV secolo. Sul personaggio vd. pure D'Arms 1976, p. 404.

15. Meiggs 19732, p. 338: " [il pontifex] is assisted in the cult of Vulcan by three praetors and two (possibly three) aediles".

16. Vidman 19822, fragm. Fa dx., a. 91 : in [fundo?] / Volusiano arb[os ful]/mine icta; cond[itum per] / aedilicios. Che si riferisca ai sacerdoti minori di Vulcano è opinione di Degrassi, I.It., XIII.1, p. 222 (ad a. 91), condivisa da Vidman 1957, p. 47; 
Come i pontifices, sono noti da numerose iscrizioni; assenti nei Fasti della colonia, li troviamo invece menzionati in liste loro riservate: Fasti anche questi ovviamente, ma diversi e distinti dagli altri. È proprio da questi che vorrei partire.

La natura di tali documenti è risultata chiara dopo la pubblicazione di una nota di A. Licordari, che nel 1984 ha fatto conoscere un gruppo di frammenti, ricongiunti da F. Zevi, contenenti l'elenco dei nomi degli edili e dei pretori degli anni 272-276 d.C. ${ }^{17}$ (fig. 1).

Alcuni anni più tardi, G. Mennella ha riconosciuto in un frammento murato in una villa privata a Punta Zancale presso Marina di Camerota (SA) un'ulteriore porzione di elenco relativa agli anni 222-229 d.C. ${ }^{18}$ (fig. 2) ed ha ricongiunto alla serie un trascurato frammento, già edito in CIL, XIV, che Dessau, senza seguito, aveva correttamente attribuito al collegio di Vulcano: Mennella, oltre a migliorarne la lettura, lo ha ricondotto agli anni 192-193 d.C. ${ }^{19}$ (fig. 3).

Vidman 19822, p. 82. Come mi fa osservare F. Zevi, si può dubitare di tale interpretazione alla luce della giovane età di coloro che talora rivestivano la carica.

17. Licordari 1984, p. 347-349, tav. LXVI (curiosamente sfuggito ad $A E$ ): dodici frammenti, di cui due perduti (nove per Licordari), ricongiunti, di una lastra in marmo bianco con venature grigie (lunense?); lacune in alto e in basso (si conservano i margini laterali originali e l'angolo inferiore destro); fronte liscia, ma molto corrosa a sinistra a causa della lunga esposizione ad agenti atmosferici; retro liscio; (99) x 90 x 3/3,8; lett. 2,6 [rr. 1-2]; 2,8 [rr. 3-6]; 2,6 [rr. 7-8];2,3 [r. 9]; 2,5 [r. 10]; 2,6 [r. 11]; 2,3 [r. 12]; 2,2 [rr. 13-14]; 2,4 [r. 15]. L'altezza delle lettere è molto irregolare anche all'interno di una stessa riga. Interlinea: 0,7 [rr. 3-6]; 0,9 [rr. 7-10]; 1,4 [rr. 10-11]; 0,9 [rr. 11-12]; 0,7 [rr. 12-13]; 0,9 [rr. 13-14]; 0,7 [rr. 14-15]. Ritrovata tra il luglio ed il dicembre 1939 nella zona di via degli Augustali (regio V): $G d S$, II, p. 166 n $^{\circ} 228$; p. 168 n $^{\circ} 235 ;$ p. 172 n $^{\circ} 234$; III, p. $34 \mathrm{n}^{\circ}$ 313. Si conserva ad Ostia, via Tecta. Inv. 6354. Autopsia 19/03/2013. Desidero ringraziare Filippo Marini Recchia per la splendida fotografia, che migliora la lettura.

18. Mennella 1995, p. 95-101, cfr. $A E$ 1995, 244: frammento di lastra in marmo bianco mutilo su tutti i lati tranne a sinistra; fronte liscia, retro murato; $21 \times 16,2 \times 1,8$ (spessore emergente); lett. 2,3-2,7. Provenienza ignota. Autopsia foto.

19. CIL, XIV 4138: frammento di lastra di marmo bianco, mutilo su tutti i lati, tranne a sinistra, dove tuttavia è scheggiato; fronte liscia; retro liscio con tracce di ruggine e leggermente rientrante; 17 x 21 x 3,2; lett. 1,6. Interlinea: 1. Trovato negli scavi condotti nella zona tra il teatro ed il tempio che è al centro del c.d. Piazzale delle Corporazioni. Si conserva ad Ostia, deposito 20, scaffale 57A. Inv. 8188 . Autopsia 19/03/2013. Dessau (non Wickert) non vide l'originale, ma si fidò di una lettura fatta da Gatti sulla base di una trascrizione di Lanciani : Lanciani 1886, p. 83.

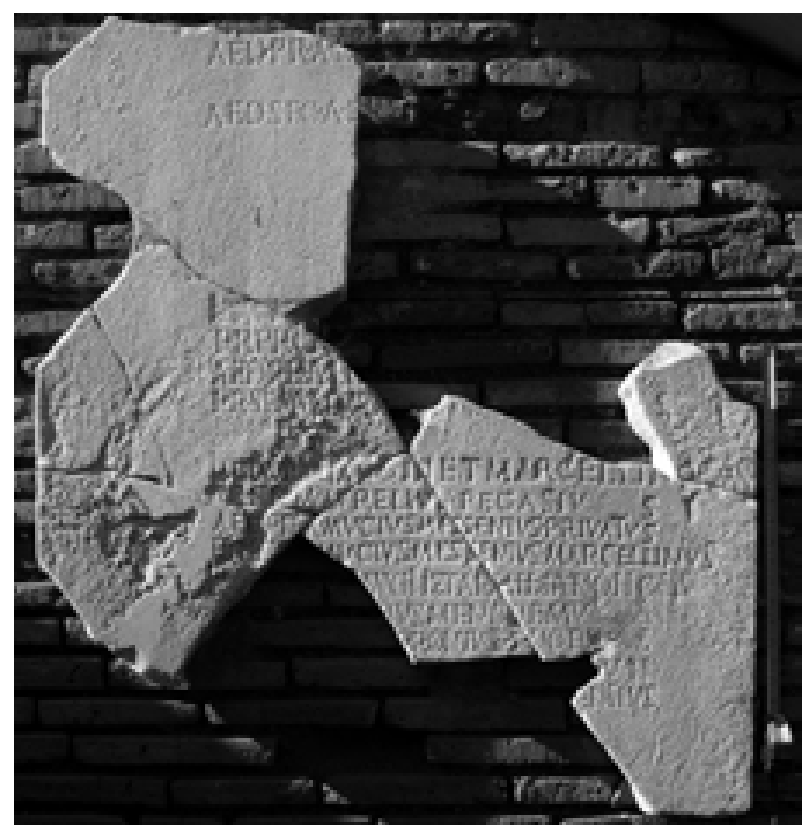

Fig. 1 - Ostia, via Tecta, inv. 6354 : frammenti della lastra con i fasti degli edili e dei pretori di Vulcano degli anni 272-276 d.C. Da Licordari 1984, p. 347-349, Concessione Soprintendenza speciale per i beni archeologici di Roma.

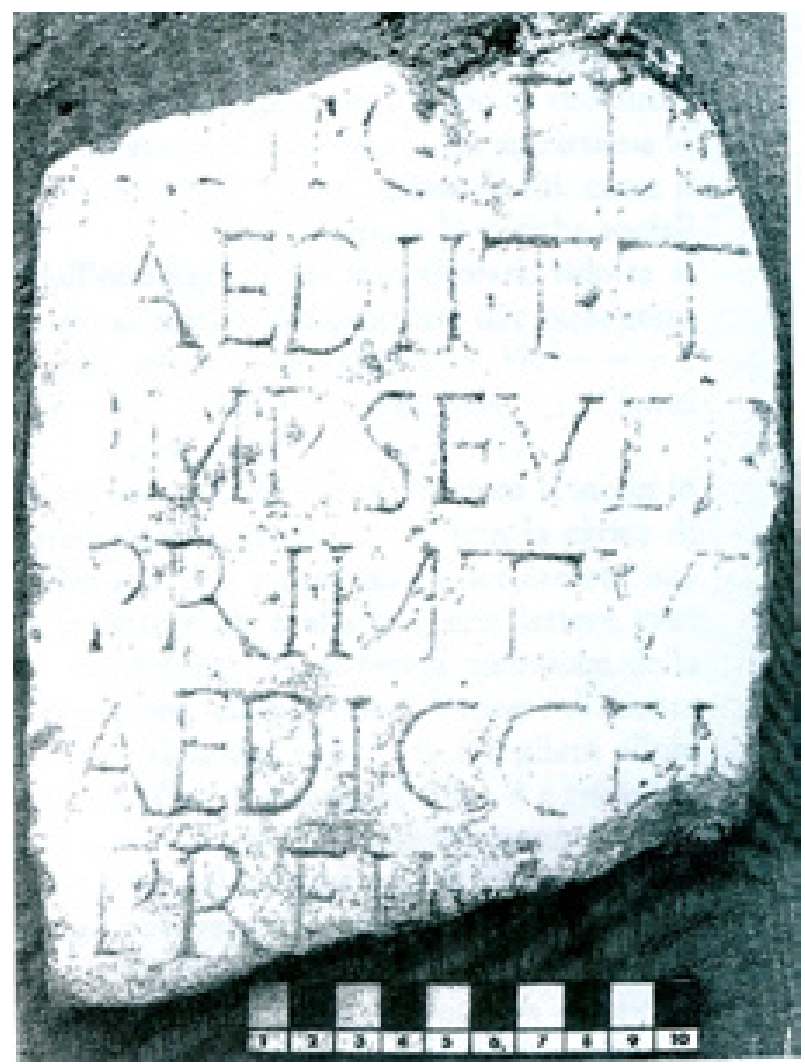

Fig. 2 - Villa privata a Punta Zancale presso Marina di Camerota (SA) : frammento della lastra con i fasti degli edili e dei pretori di Vulcano degli anni 222-229 d.C.

Da Mennella 1995, p. 96. 


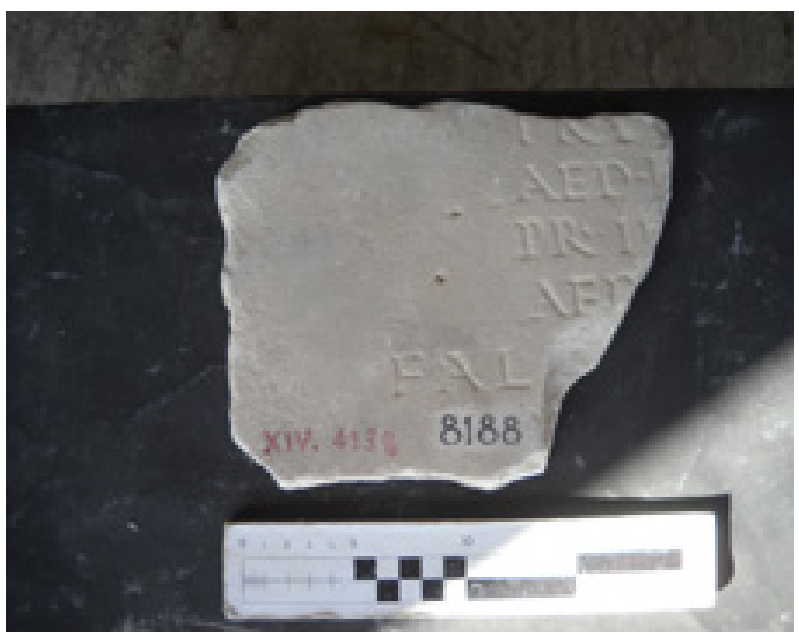

Fig. 3 - Ostia, deposito 20, inv. 8188 : frammento della lastra con i fasti degli edili e dei pretori di Vulcano degli anni 192-193 d.C. CIL, XIV 4138, Concessione Soprintendenza speciale per i beni archeologici di Roma.

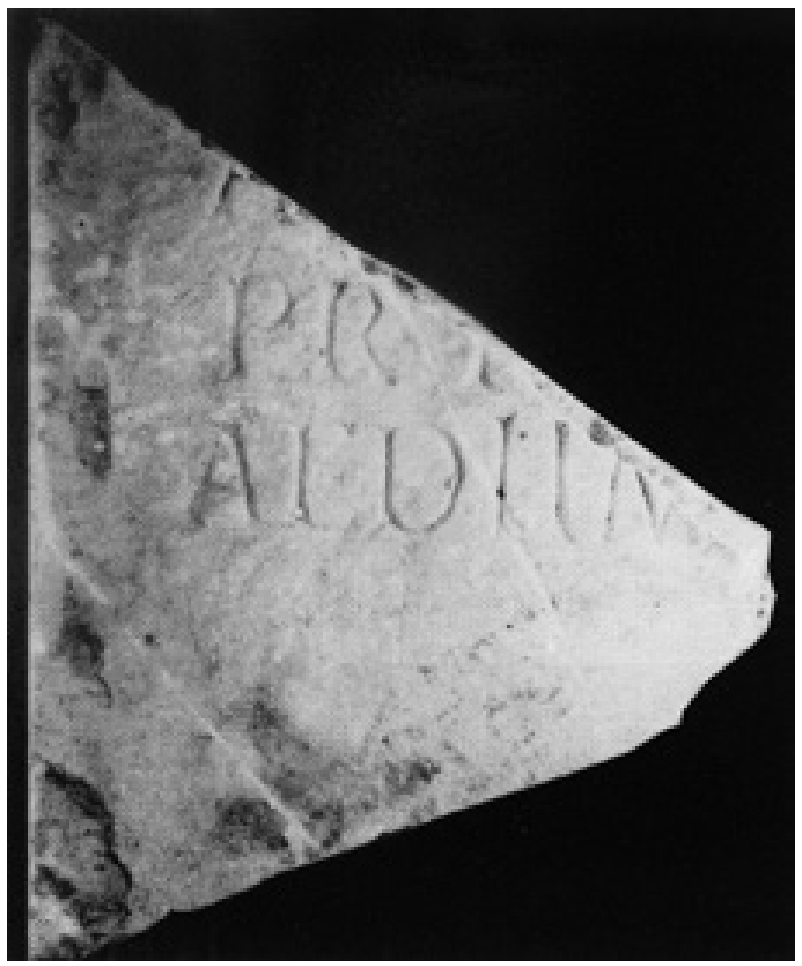

Fig. 5 - Ostia, dalla Basilica di Pianabella, deposito 20, inv. 36438 : frammento di lastra con i fasti degli edili e dei pretori di Vulcano di anno incerto.

AE 2001, 634 d, Concessione Soprintendenza speciale per i beni archeologici di Roma.

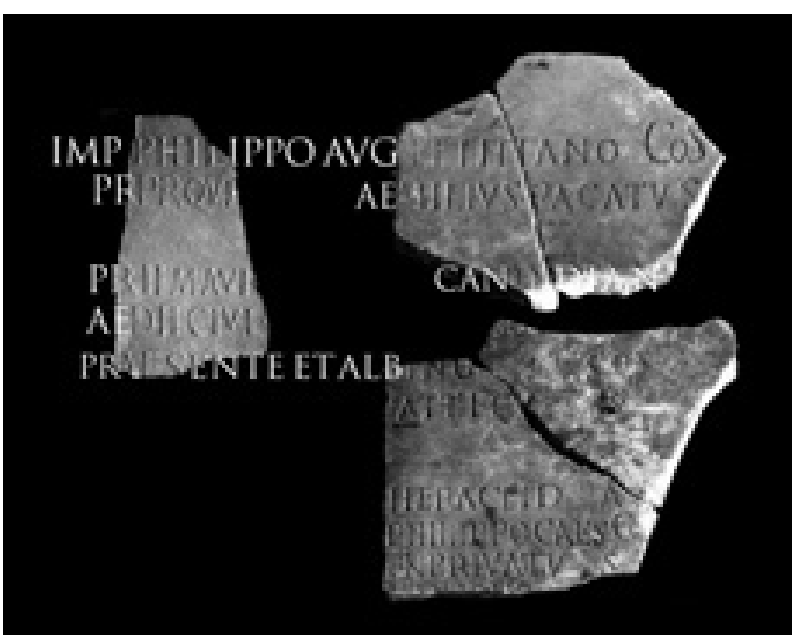

Fig. 4 - Ostia, dalla Basilica di Pianabella, invv. 48909, 47817, $47799+47898$ (irreperibili) : frammenti della lastra con i fasti degli edili e dei pretori di Vulcano degli anni 245-247 d.C.

AE 2001, 634 a-c, Concessione Soprintendenza speciale per i beni archeologici di Roma.

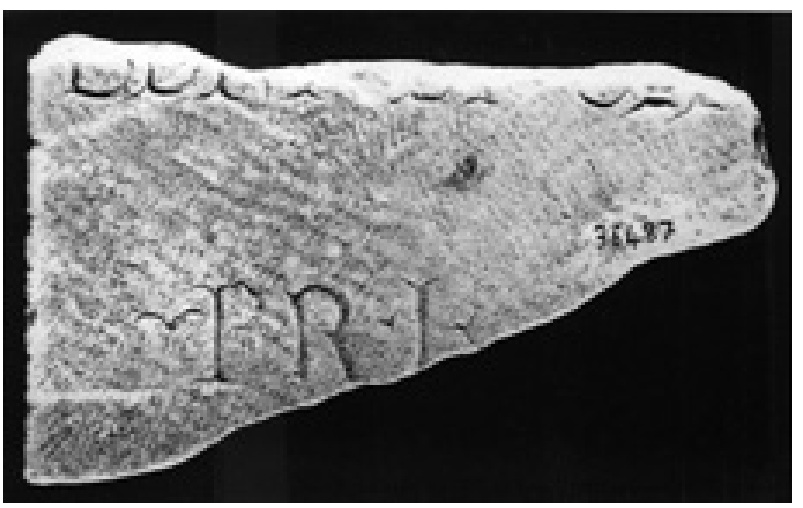

Fig. 6 - Ostia, dalla Basilica di Pianabella, deposito 20, inv. 36487 frammento di lastra con i fasti degli edili e dei pretori di Vulcano di anno incerto.

AE 2001, 634 e, Concessione Soprintendenza speciale per i beni archeologici di Roma.

La recente pubblicazione di alcune iscrizioni provenienti da Pianabella ha fatto conoscere altri sette frammenti, cinque dei quali, contigui, si riferiscono agli anni 245-247 d.C., mentre gli altri due non sono databili con precisione ${ }^{20}$ (figg. 4-6).

20. Nuzzo 1999, p. 40-42 n A7, cfr. $A E$ 2001, 634 a-e. A: frammento interno di lastra marmorea; fronte e retro lisci $22 \times 13 \times 5$; lett. 2,5. Dall'atrio della basilica. Inv. 48909. Irreperibile 2013. Autopsia foto. - B : due frammenti ricongiunti di lastra marmorea, mancanti su ogni lato; fronte e retro lisci; 20 x $27 \times 4$; lett. 3,5-2. Inv. 47817. Irreperibile 2013. Autopsia foto. - C : due frammenti ricongiunti di lastra marmorea mancanti su ogni lato; fronte e retro lisci; $22 \mathrm{x}$ 32 x 4; lett. 3,5-2. Invv. $47799+47898$. Irreperibile 2013. 
Fornisco di seguito una nuova trascrizione degli elenchi, migliorativa in alcuni punti, con una disposizione in ordine cronologico:

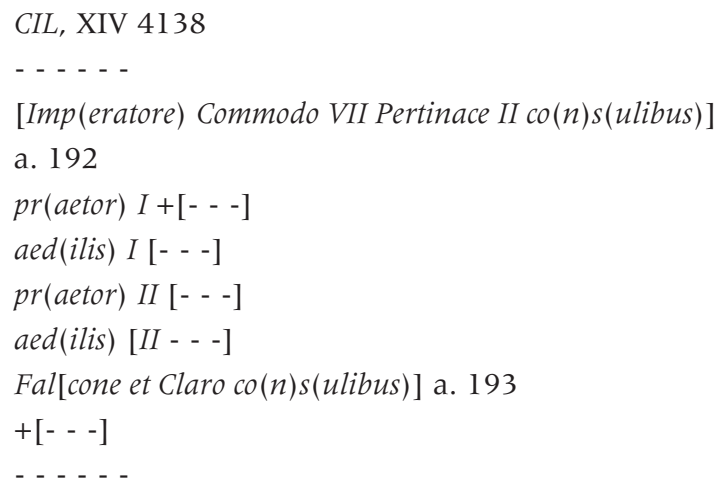

Le parole sono regolarmente separate da punti. R. 7: tratto di graffia in margine di frattura non segnalata da Mennella. Si notino l'ampio margine a sinistra e l'indicazione consolare sporgente a sinistra.

$A E$ 1995, 244

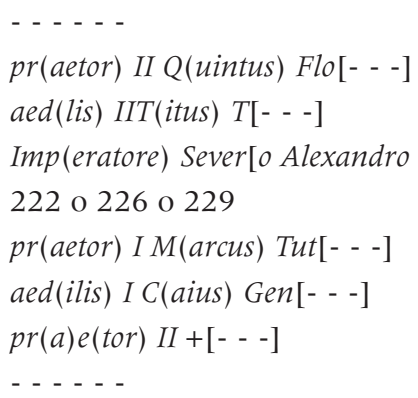

Segni di interpunzione tra le parole. R. 1: $E$ sulla pietra; r. 6: tra le soluzioni prospettate da Mennella preferisco quella qui presentata, che non esclude una errata interpretazione del numerale II

\footnotetext{
Autopsia foto. - D : frammento di lastra marmorea di cui si conserva il margine sinistro; fronte gradinata, retro liscio; $24 \times 18,5 \times 5,2$; lett. 2,5; interlinea: 1,5 e 1,3; vacat: 9,5. Dai colombari Q. 13-14/ A-B. N. Inv. 36438. Si conserva ad Ostia, deposito 20, scaffale 104D, cassetta gialla. Autopsia 25/9/2013. - E: frammento di lastra marmorea di cui si conserva il margine sinistro; fronte gradinata, retro liscio; $14 \times 24,5 \times 3,2$; lett. 3,2; vacat: 6,3. Inv. 36487. Si conserva ad Ostia, deposito 20, scaffale 107D. Autopsia 25/9/2013. A-C sono pertinenti alla stessa lastra: la ricomposizione grafica è di Filippo Marini Recchia, che, ancora una volta, ringrazio. La lastra doveva avere a sinistra uno spessore di $5 \mathrm{~cm}$ che diminuiva progressivamente verso destra, dove arrivava ad avere uno spessore di $4 \mathrm{~cm}$.
}

come una forma di $E$ corsiva. Si notino lo scarso margine a sinistra e l'indicazione consolare allineata a sinistra con l'elenco dei sacerdoti.

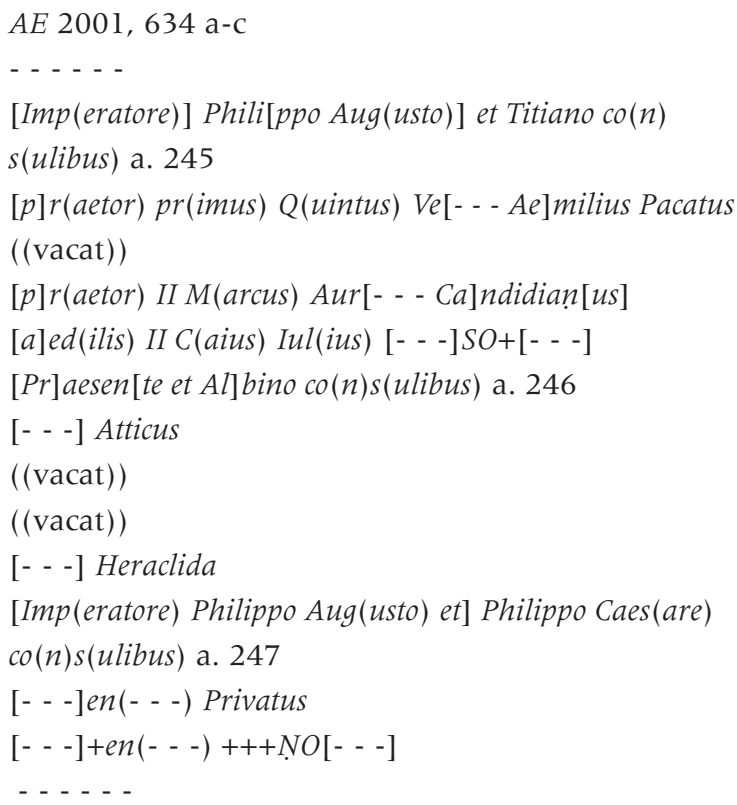

La precedente trascrizione non tiene conto dei vacat, importanti per comprendere la struttura del testo. Allineamento a destra della lettera finale di nome. Dalla ricostruzione risulta che l'indicazione consolare doveva sporgere a sinistra, soprattutto in corrispondenza del nome dell'imperatore. R. 3: [Ca]ndidian $[u s]$ o [Ca]ndidian(us). Il gentilizio Aurelius poteva essere abbreviato e seguito da un altro elemento onomastico oppure scritto per esteso. R. 4: C o G.

Licordari 1984, P. 347-349

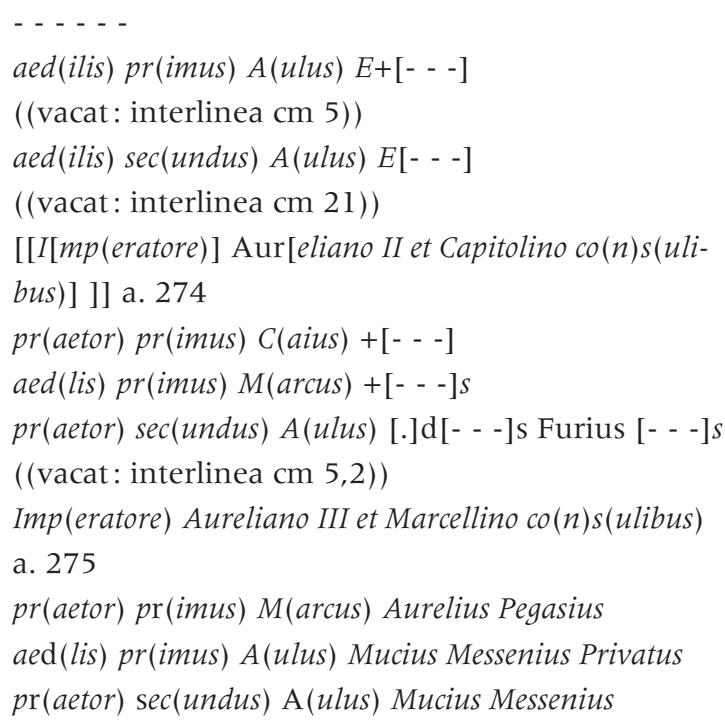




\author{
Marcellinus \\ Imp(eratore) Tacito Aug(usto) II et Aemiliano II \\ $\operatorname{co}(n) s($ ulibus) a. 276 \\ [pr(aetor) pr(imus) - - - ]cius Menedemus \\ [aed(lis) pr(imus) - - -]pronius Zenobius \\ [pr(aetor) sec(undus) - - -] iun(ior) \\ [aed(ilis) sec(undus) - - - ]entius \\ ((vacat fino a fine lastra))
}

R. 1: A.E[grilius? ? - - -] Licordari; r. 2 : A.E[grilius??

- - - Licordari; r. 3 : erasione non segnalata da

Licordari; r. 4 fin. : resto di tratto verticale non

segnalato da Licordari; r. 5 : [-] V[- - - ]s Licordari;

r. 6 : la parte finale non è più leggibile, sia perché

evanida, sia perché è probabilmente andato perduto

un frammento come lascerebbe supporre il chiodo

sporgente lungo il margine di frattura; r. $7: I$ in.

montante; r. 9: T montante in Privatus; r. 11 : il

nome Tacito manca per perdita di un frammento, lo

stesso che nella riga precedente doveva contenere il praenomen Aulus; r. 13: [?Ze]nobius Licordari.

Oltre alle difformità segnalate, la trascrizione del primo editore non tiene conto dei vacat e non segnala l'ampio spazio vuoto dopo l'ultima riga. Inoltre: sono da notare la diversa grafia nella abbreviazione cos. e nelle rr. 6-7, 8-9 e 12-15 l'allineamento a destra della lettera finale; l'ampio margine a sinistra e l'indicazione consolare allineata a sinistra con l'elenco dei sacerdoti.

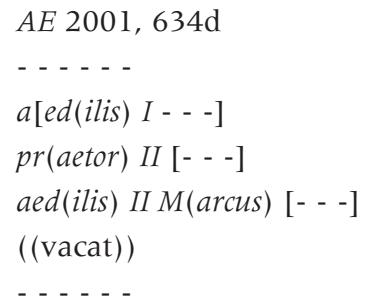

La precedente trascrizione a r. 2 non vede il piede del secondo numerale e non registra l'ampio spazio vuoto dopo l'ultima riga. Si noti lo scarso margine a sinistra.

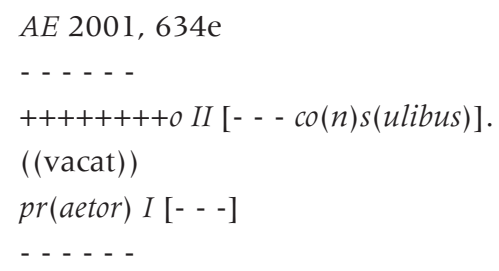

Uso regolare di interpunti. L'impaginazione di r. 1 , che sporge a sinistra, unitamente alla menzione a r. 2 del praetor I, lascia supporre che qui dovesse trovarsi la notazione consolare. Il segno curvo della terz'ultima lettera e le tracce di segni verticali ravvicinati per penultima e ultima lettera suggeriscono la lettura proposta.

Ritengo che possano essere aggiunti alla serie altri due frammenti, il primo con qualche dubbio, il secondo con certezza, già editi, ma non riconosciuti nella loro natura.

Il primo, pubblicato in CIL, XIV 4137, è un frammento di lastra di marmo bianco (lo stesso di CIL, XIV 4138), mutilo su tutti i lati, tranne a destra, dove si conserva il margine originale; la fronte è liscia; il retro è liscio con tracce di ruggine. Misura 14 x 15 x 3,5; lett. 1,5 (ma 0,9 la $O$ di cos.). Interlinea: 0,8 [rr. 1-4], 1,5 [rr. 4-5]. Fu trovato, insieme con CIL, XIV 4138 (anche se i due frammenti non possono appartenere alla stessa lastra), negli scavi condotti nella zona tra il teatro ed il tempio che è al centro del c.d. Piazzale delle Corporazioni. Si conserva ad Ostia (deposito 20, scaffale 57A. Inv. 8187. Autopsia 19/03/2013). Anche in questo caso, Dessau non vide l'originale, ma utilizzò una lettura fatta da Gatti sulla base di una trascrizione di Lanciani (fig. 7).

$$
\begin{aligned}
& ----- \\
& {[---] u[s]} \\
& {[---] \text { rius }} \\
& {[---] x} \\
& {[-- \text { - } n u s} \\
& {[-- \text { - Augg.(i.e. Augustis duobus) co(n)s(ulibus) }} \\
& -----
\end{aligned}
$$

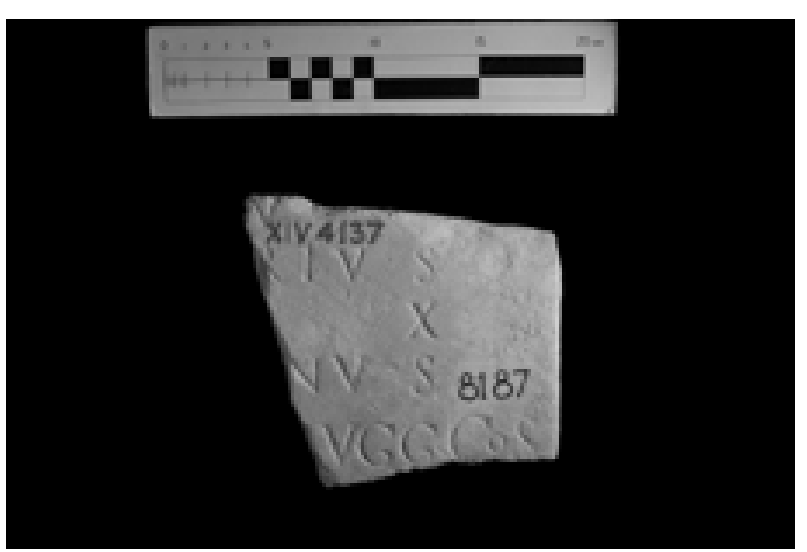

Fig. 7 - Ostia, deposito 20, inv. 8187: frammento di lastra forse relativo ai fasti degli edili e dei pretori di Vulcano di anno incerto. CIL, XIV 4137, Concessione Soprintendenza speciale per i beni archeologici di Roma. 


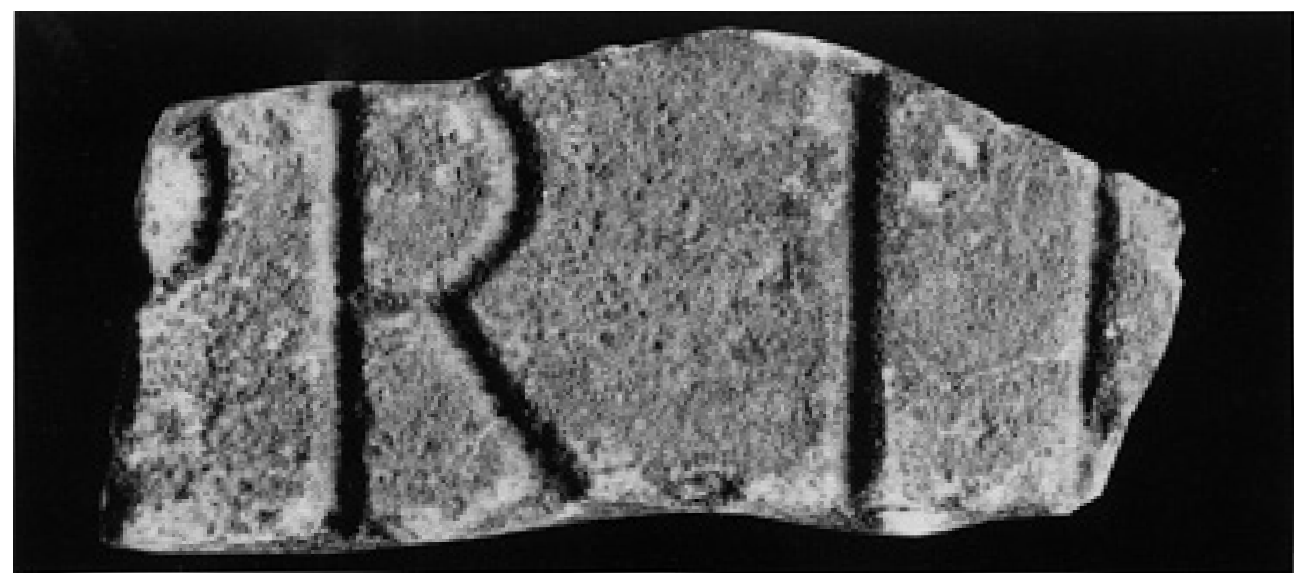

Fig. 8 - Ostia, dalla Basilica di Pianabella, inv. 48931 (irreperibile) : frammento di lastra con i fasti degli edili e dei pretori di Vulcano di anno incerto.

Nuzzo 1999, p. $40 n^{\circ}$ A6, Concessione Soprintendenza speciale per i beni archeologici di Roma.

Il secondo documento, pubblicato nello stesso volume su Pianabella che ha reso noti i Fasti degli anni $245-247^{21}$, è un frammento di lastra in marmo bianco mutilo su tutti i lati con retro liscio. Misura 6 × 13 x 4. Fu trovato nel 1989 a Pianabella, nell'area stradale a nord della basilica (irreperibile 2013; inv. 48931) (fig. 8).

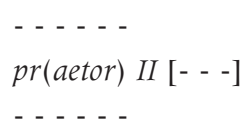

Il dossier venutosi a creare negli ultimi trent'anni impone alcune considerazioni. Come si è visto, sono noti i fasti dall'anno 192 al 276, sia pur in stato terribilmente frammentario. Tuttavia, se la sola sequenza relativa all'elenco di metà dei componenti del collegio dell'anno 272 fino a quello completo dell'anno 276 sviluppa un'altezza non completa di $99 \mathrm{~cm}$, dobbiamo di necessità supporre che anche i Fasti degli edili e dei pretori di Vulcano, al pari dei Fasti della colonia, si articolassero in più lastre. Queste inoltre, a giudicare sempre dall'esemplare più completo (quello degli anni 272-276), dovevano avere una larghezza non inferiore ai $90 \mathrm{~cm}$ (circa tre piedi).

Ricordo come utile confronto che i Fasti della colonia erano disposti su almeno 19 lastre alte

21. Nuzzo 1999, p. $40 \mathrm{n}^{\circ}$ A6, che leggeva ed interpretava, sia pur in via ipotetica, $\operatorname{pr}$ (aefectus) $i$ (ure) $d$ (icundo).
150/220 cm e larghe $70 \mathrm{~cm}$ (sviluppo complessivo: $13,30 \mathrm{~m})$.

Le lastre dei Fasti degli edili e dei pretori di Vulcano dovevano tappezzare per un ampio spazio un edificio (o una sua pertinenza), che, a ragione, è stato supposto essere il tempio di Vulcano ${ }^{22}$.

Il fatto che la più antica notazione si riferisca all'anno 192 nulla ci dice sulla data di inizio della compilazione, che può ben risalire a quegli anni e dunque essere un portato delle trasformazioni istituzionali dell'età tardoantonina, ma che può

22. Come ho detto all'inizio, non è mia intenzione affrontare il problema della localizzazione del culto. Ricordo tuttavia che due frammenti dei Fasti degli edili e dei pretori di Vulcano (CIL, XIV 4138, di sicura pertinenza, relativo agli anni 192-193 e CIL, XIV 4137, di incerta pertinenza e di anno ignoto) sono stati ritrovati nella zona tra il teatro ed il tempio che è al centro del c.d. Piazzale delle Corporazioni. Dagli scavi del teatro (Reg. II, is. VII, cas. 2), ma potrebbe trattarsi di un reimpiego, viene gran parte dei frammenti che compongono CIL, XIV 4724, una lastra di rivestimento di architrave in cui è menzione di un intervento in relazione ad una [- - ae]dem Vo[lc]ano. Pellegrino 1986, p. 301 lascia la questione nel vago, ma Coarelli 1994, p. 42-43 trae come conclusione che il tempio al centro del c.d. Piazzale delle Corporazioni dovesse essere identificato con quello di Vulcano. Per l'ipotesi che anche i Fasti della colonia fossero esposti nel tempio di Vulcano vd. Vidman 1957, p. 86 s.; ma cfr. Vidman 19822, p. 148. I Fasti degli edili e dei pretori di Vulcano non annotavano i pontefici del culto, registrati invece nei Fasti della colonia. L'osservazione è già in Licordari 1984, p. 348. Mennella 1995, p. 99 la spiega per il fatto che la carica era vitalizia, laddove i fasti erano per loro natura annuali. La spiegazione non convince, poiché anche i Fasti della colonia erano annuali. Possiamo solo dire che nei frammenti conservati non ce ne è menzione. 
anche precedere, e di molto, la fine del II secolo. Parimenti il fatto che la notazione più recente sia riferita all'anno 276 non significa che la redazione dei Fasti cessasse con l'ultimo quarto del III secolo. Sappiamo da altra fonte, come si è visto, che il pontificato di Vulcano doveva essere ancora vivo in età dioclezianea ${ }^{23}$ e dunque il culto di Vulcano ancora praticato.

Indipendentemente dall'epoca di inizio della loro notazione, dobbiamo comunque immaginare che i Fasti degli aediles e dei praetores di Vulcano avessero un sistema di redazione "aperto", al pari dei Fasti della colonia. Questo spiega la mancanza di uniformità sotto il profilo paleografico, mentre non spiega del tutto la scelta di indicare con il numerale o con l'iniziale abbreviata la posizione all'interno del collegio: significativo è in questo senso il frammento pertinente all'anno 245, con la menzione del praetor pr(imus) e del praetor II. Tuttavia, pur nella lacunosità della documentazione, andrà rilevato come nei frammenti relativi agli anni 192-193 e in quelli del 222 o 226 o 229 la specificazione numerica sia espressa sempre con la cifra, mentre nel frammento relativo agli anni 272-276 sia sempre espressa con il numerale abbreviato. È dunque possibile che intorno alla metà del III secolo, dopo un periodo in cui è stato sperimentato un sistema di indicazione misto, si sia optato per un sistema diverso da quello della fine del II-prima metà del III secolo. Questa osservazione potrebbe servire per cercare di collocare in una serie cronologica i frammenti che non contengono la data consolare.

Altra questione è quella delle fasi di redazione. Il frammento relativo agli anni 192-193 sembrerebbe vedere all'opera la mano di uno stesso lapicida e lo stesso sembra potersi dire per gli anni 222 o 226 o 229 e precedente. Al contrario, nell'insieme di frammenti che ricompongono la sequenza degli anni 245-247 e 272-276 sembra di vedere un cambio di mano (si noti il diverso modo di scrivere l'abbreviazione cos.). Resta comunque il fatto che, come ha osservato Vidman per i Fasti della colonia ${ }^{24}$, non necessariamente le differenze di ductus sono prova di fasi di redazione cronologicamente diverse, potendo essere impiegati più scalpellini per attendere all'incisione del testo. La questione è tuttavia di una certa rilevanza non solo per conoscere la storia interna del documento, ma anche per cercare di capire il senso dei vacat presenti in alcune liste. Per l'anno 245 la riga in cui avrebbe dovuto trovare posto il nome dell'aedilis I è vuota; per l'anno 246 restano vuote le righe relative all'aedilis I e al praetor II; per l'anno 274 resta vuota la riga relativa all'aedilis II; l'ampio vacat $(21 \mathrm{~cm})$ che precede la datazione consolare del 274 avrebbe potuto ospitare la serie di un intero anno (il 273), mentre il vacat tra le righe 2-3 avrebbe potuto ospitare l'indicazione del praetor sec(undus). Già Mennella si era chiesto se tali collegialità incomplete "fossero intenzionali o dipendessero da una concreta impossibilità di completarle da parte degli organi responsabili $»^{25}$. Senza poter escludere questa soluzione, alla luce delle considerazioni fatte, mi chiedo invece se ciò non sia legato ad una questione redazionale, vale a dire, nel caso di una redazione a posteriori, se il vacat non derivi dalla mancanza di documentazione proveniente dagli archivi e messa a disposizione dei redattori.

Quello che invece, anche alla luce dei nuovi frammenti, sembra confermato è che l'organico del collegio dovesse essere formato da due praetores e da due aediles: la carica di praetor tertius, attestata solo per P. Lucilius Gamala Iunior resta un unicum $^{26}$, al pari del fatto di essere stato anche prima aedilis e poi pontifex del culto (l'unico lontano confronto è quello con il suo omonimo antenato, che fu però solo aedilis e pontifex: vd. infra). Più complessa è la questione della gerarchia interna e della possibilità di un cursus. La struttura dei Fasti sembra alludere ad una gerarchia di importanza; quello che non è chiaro è se i praetores nel loro insieme siano da considerarsi più importanti degli aediles (così sembrerebbe dal frammento relativo al periodo che precede l'anno 274, dove troviamo in successione aed(ilis) pr(imus) e aed(ilis) sec(undus): tuttavia la lacuna iniziale e i vacat impediscono di sapere se i praetores fossero indicati) oppure, più probabilmente, ferma restando la superiorità del pretore rispetto all'edile, se la discriminante fosse nell'attributo primus e secundus (in tal caso l'aedilis primus sarebbe gerarchicamente superiore al
23. Vd. supra nota 14.

24. Cfr., ad esempio, Vidman 19822, frr. Ca 1-5 e Qa - Qb rr. 1-9, 14-20.
25. Mennella 1995, p. 101.

26. Diversamente Nuzzo 1999, p. 41. 
praetor secundus). Nulla invece sembra parlare in favore di un cursus interno al collegio. Né i Fasti, né la restante documentazione sembrano andare in questa direzione. Infatti, se si escludono i casi, peraltro peculiari, dei due P. Lucilius Gamala, Senior e Iunior, che furono rispettivamente aedilis e pontifex l'uno, aedilis, praetor tertius e poi pontifex l'altro, resta solo Cn. Turpilius Turpilianus, che una dedica sacra ricorda quale aedilis e praetor del culto. D'accordo con Meiggs ${ }^{27}$ e contro Carcopino ${ }^{28}$, sembra dunque esistere una gerarchia ma non una propedeuticità. Di norma si ricopriva una sola carica, quella di aedilis o quella di praetor, e né l'una né l'altra costituivano premessa per diventare pontifex.

Se i Fasti ci danno conferma della annualità della carica ${ }^{29}$, nulla ci dicono in merito alle modalità di nomina. Qualche nuovo dato viene invece dalle iscrizioni. Se l'iscrizione relativa a M. Marius M.f. Pal. Primitivus poteva lasciare dubbi riguardo l'interpretazione dell'espressione decur. dec. aed. II sac. V[olk. $]^{30}$, perspicui sono invece i casi di $N$. Trebonius N.f. Venerius, che dice $[d]$.$d . aed [i l i] s$ allectus sa[c. Volc. f. $]^{31}$ e soprattutto di A. Egrilius A.f. Pal. Magnus, che pure dice, ma questa volta senza lacune, dec. decr. praet. primus sacris Volk. faciundis $^{32}$. Oggi, dunque, a differenza di quando Mennella scriveva, l'iscrizione di M. Marius M.f. Pal. Primitivus non è più l'unica a riportare la prassi di una nomina decurionale dei sacerdoti minori di Vulcano e con maggiore difficoltà di un tempo si può parlare, almeno per il II secolo, di un iter del tutto straordinario ${ }^{33}$.

Sono ancora le iscrizioni a consentirci qualche ulteriore considerazione sulla condizione sociale degli edili e dei pretori di Vulcano.

Per una disamina del problema, possono essere di qualche utilità le tabelle che seguono, con le quali, tra l'altro, si aggiorna l'elenco fornito da Mennella degli edili e dei pretori conosciuti ${ }^{34}$ (i nuovi sono indicati in grassetto), cercando per quanto possibile di datare i personaggi e di disporli in ordine cronologico. Un asterisco segnala coloro che furono eccezionalmente sia aedilis sia praetor del culto:

\begin{tabular}{|c|c|c|c|c|}
\hline \multicolumn{5}{|l|}{ AEDILES } \\
\hline $\begin{array}{l}\text { 1) } C I L, \mathrm{XIV} 375= \\
\text { ILS } 6147\end{array}$ & $\begin{array}{l}\text { P. Lucilius Gamala Senior } \\
\text { Vd. Gallina Zevi - Humphrey } \\
\text { 2004, p. } 47-98 \text { (con interventi di } \\
\text { F. Zevi, S. Panciera, M. Cébeillac- } \\
\text { Gervasoni, E. Lo Cascio, F. } \\
\text { Coarelli), dove si fa il punto della } \\
\text { situazione negli studi sul perso- } \\
\text { naggio. }\end{array}$ & aed. sacr. Volk. (poi pontifex) & $\begin{array}{l}\text { I sec. } \\
\text { a.C., } \\
\text { metà }\end{array}$ & $\begin{array}{l}\text { aedilis, decurio, IIvir c. } \\
\text { p. q., curator pecuniae } \\
\text { publicae exigendae et } \\
\text { adtribuendae }\end{array}$ \\
\hline $\begin{array}{l}\text { 2) } C I L, \mathrm{XIV} 351= \\
I L S 6145\end{array}$ & $\begin{array}{l}\text { A. Fabius A.f. Pal. Felicianus } \\
\text { Meiggs } 1973^{2} \text {, p. } 338 \text {. CIL, XIV } \\
351 \text { è un frammento di sarcofago. } \\
\text { Si conserva ad Ostia, via Tecta, } \\
34 \text { x } 126 \text { x } 7 \text { : inv. } 11009 \text {. }\end{array}$ & aedilis sacr. Volk. $f$. & II sec. & $\begin{array}{l}\text { morto a } 19 \text { anni; non } \\
\text { ricopre altre cariche }\end{array}$ \\
\hline
\end{tabular}

27. Meiggs 19732, p. 338.

28. Carcopino 1919, p. 44 e 65.

29. Meiggs 19732, p. 338, che non conosceva i frammenti dei Fasti, scriveva: «It seems likely that the office was held for one year only...".

30. CIL, XIV 4553; cfr. $A E 1989,125$, che è una seconda copia della precedente: la carica è qui però integrata. L. Wickert, ad CIL, ricorda, senza condividerla, l'integrazione e lettura di Carcopino 1919, p. 65 : [d(ecreto)] / decur(ionum) dec(urio), aed(ilis) II sac. V[olk.].
31. CIL, XIV 5379 + Marinucci 1992, p. 200-201 n C64.

32. Sijpesteijn 1996, p. 286-288 n 7, cfr. $A E 1996,304$ a-b.

33. Si osservi che, sempre per il II secolo, ad Ostia, la nomina decurionale compare anche per le magistrature ordinarie. Resta naturalmente la questione se nel II secolo effettivamente l'ordo decurionum abbia acquisito un'importanza maggiore del passato, a detrimento di altre istituzioni (possibile), o, ovviamente, che proprio la notazione sottolinei un iter fuori norma. E' vero che i casi su cui riflettere sono comunque troppo pochi. Sulla questione vd. Caldelli 2008, p. 270-272.

34. Mennella 1995, p. 100 nota 16. 


\begin{tabular}{|c|c|c|c|c|}
\hline $\begin{array}{l}\text { 3) } C I L, \text { XIV } 4553, \\
\text { cfr. } A E 1989,125\end{array}$ & $\begin{array}{l}\text { M. Marius M.f. Pal. Primitivus } \\
\text { Meiggs } 1973^{2}, \text { p. } 178 \text { (numero } \\
\text { degli edili), } 325 \text { (cfr. CIL, XIV } \\
\text { 4554; 5327-5328?); D'Arms } \\
\text { 1976, p. 408-409. }\end{array}$ & decur. dec. aed. II sac. V[olk.] & $\begin{array}{l}\text { a. } 145 \\
\text { d.C. }\end{array}$ & $\begin{array}{l}\text { non ricopre altre } \\
\text { cariche }\end{array}$ \\
\hline $\begin{array}{l}\text { 4) } C I L, \text { XIV } 3=I L S \\
3299\end{array}$ & $\begin{array}{l}\text { *Cn. Turpilius Cn.f. Turpilianus } \\
\text { Meiggs } 1973^{2}, \text { p. } 178,338 \text {. CIL, } \\
\text { XIV } 3 \text { è perduta. Turpilius è genti- } \\
\text { lizio raro ma attestato ad Ostia, } \\
\text { cfr. CIL, XIV 247, III. 11, data- } \\
\text { bile agli anni 139-145 d.C. dove } \\
\text { compare un Cn. Turpilius (altre } \\
\text { due attestazioni sono CIL, XIV } \\
\text { 5128/29 e forse 4826). }\end{array}$ & aedil. et pr. sac. Volk. fac. & $\begin{array}{l}\text { II sec., } \\
\text { prima } \\
\text { metà (?) }\end{array}$ & $\begin{array}{l}\text { non ricopre altre } \\
\text { cariche }\end{array}$ \\
\hline $\begin{array}{l}\text { 5) } C I L, \text { XIV } 4641+ \\
4644=A E 1986 \\
113\end{array}$ & $\begin{array}{l}\text { A. Egrilius Paternus } \\
\text { Scheid 1999, p. } 142 .\end{array}$ & aedilis sacr. Volk. $f$. & $\begin{array}{l}\text { II, prima } \\
\text { metà (?) }\end{array}$ & $\begin{array}{l}\text { figlio di liberti; eq. } R ., \\
\text { lictor curiatius, flamen } \\
d[i v] i \text { Vesp. }\end{array}$ \\
\hline 6) $C I L$, XIV 376 & $\begin{array}{l}\text { *P. Lucilius Gamala Iunior } \\
\text { Meiggs } 1973^{2}, \text { p. } 178,185,338, \\
495-496 .\end{array}$ & $\begin{array}{l}\text { aed. sacr. Volcani, eiusdem pr. } \\
\text { tert. (poi pontifex) }\end{array}$ & $\begin{array}{l}\text { II sec., } \\
\text { seconda } \\
\text { metà }\end{array}$ & $\begin{array}{l}\text { decurio, IIvir praefectus } \\
\text { L. Caesar(is) Aug(usti) } \\
\text { f(ili) cens(or), quaes- } \\
\text { tor aerarii, tabular. et } \\
\text { librorum curator, curator } \\
\text { pecuniae publicae exi- } \\
\text { gendae et attribuendae }\end{array}$ \\
\hline $\begin{array}{l}\text { 7) CIL, XIV 390- } \\
391=I L S 6139\end{array}$ & $\begin{array}{l}\text { P. Nonius P.f. Pal. Livius } \\
\text { Anterotianus } \\
\text { Meiggs } 1973^{2}, \text { p. } 211 \text {. Il perso- } \\
\text { naggio è da mettere in relazione } \\
\text { con A. Livius Anteros e Livia } \\
\text { Marcellina attestati dall'iscrizione } \\
\text { pubblicata da Royden 1988, } \\
\text { p. } 242 \mathrm{n}^{\circ} 2 \text {, cfr. AE 1989, 124, e } \\
\text { con A. Livius Anteros di CIL, XIV } \\
\text { 4656. Vd. D'Arms 1976, p. } 402 \text {. }\end{array}$ & $\begin{array}{l}\text { aedilis pr. sacr. Volk. faciu. } \\
\text { Si preferisce la lettura aedilis } \\
\text { pr(imus) ad aedilis pr(aetor), } \\
\text { sia perché negli altri soli due } \\
\text { casi noti un elemento gram- } \\
\text { maticale specifica la doppia } \\
\text { carica, sia perché poco } \\
\text { si giustificherebbe l'uso } \\
\text { dell'abbreviazione proprio } \\
\text { per la carica più prestigiosa. }\end{array}$ & $\begin{array}{l}\text { II sec., } \\
\text { seconda } \\
\text { metà }\end{array}$ & $\begin{array}{l}\text { nipote di un liberto; } \\
\text { cavaliere, decurio, } \\
\text { flamen divi Hadriani, } \\
\text { salius Laurentium } \\
\text { Lavinatium }\end{array}$ \\
\hline $\begin{array}{l}\text { 8) CIL, XIV } 5379+ \\
\text { Marinucci } 1992, \\
\text { p. } 200-201 \mathrm{n}^{\circ} \mathrm{C} 64\end{array}$ & $\begin{array}{l}\text { N. Trebonius N.f. Venerius } \\
\text { Trebonius è gentilizio raro ad Ostia } \\
\text { e, accompagnato dal prenome } \\
\text { Numerius, compare in CIL, XIV } 2 \\
\text { (datata all'anno } 197 \text { d.C.), } 1183 \\
\text { e } 5066 .\end{array}$ & $\begin{array}{l}{[d .] \text { d. aed }[\text { ili }] \text { s allectus } s a[c .} \\
\text { Volc. f. }]\end{array}$ & $\begin{array}{l}\text { II sec., } \\
\text { fine (?) }\end{array}$ & $\begin{array}{l}\text { figlio di un liberto, } \\
\text { morto a XIII[---] anni; } \\
\text { non ricopre altre } \\
\text { cariche }\end{array}$ \\
\hline $\begin{array}{l}\text { 9) CIL, XIV 4138, } \\
\text { r. } 3\end{array}$ & $+[---]$ & aed. I & a. 192 & \\
\hline $\begin{array}{l}\text { 10) CIL, XIV 4138, } \\
\text { r. } 5\end{array}$ & {$[---]$} & aed. $[I I]$ & a. 192 & \\
\hline $\begin{array}{l}\text { 11) } A E \text { 1995, 244, } \\
\text { r. } 2\end{array}$ & T. T[- - - ] & aed. II & $\begin{array}{l}\text { a. } 221 \\
\text { o } 225 \text { o } \\
228\end{array}$ & \\
\hline $\begin{array}{l}\text { 12) } A E 1995,244 \text {, } \\
\text { r. } 5\end{array}$ & $\begin{array}{l}\text { C. Gen[- - - } \\
\text { Da integrarsi probabilmente con } \\
\text { Gen[icius] o Gen[ucius] in base alla } \\
\text { frequenza delle attestazioni ad } \\
\text { Ostia. È tuttavia da ricordare che, } \\
\text { in relazione a questo gentilizio, i } \\
\text { prenomi ricorrenti sono Aulus e } \\
\text { Lucius. Vd. Salomies 2002, p. } 157 .\end{array}$ & aed. I & $\begin{array}{l}\text { a. } 222 \\
\text { o } 226 \text { o } \\
229\end{array}$ & \\
\hline $\begin{array}{l}\text { 13) } A E 2001,634 \\
\text { a-c, r. } 4\end{array}$ & $C($ aius $) \operatorname{Iul}(\mathrm{ius})[---] S O+[---]$ & [a]ed. II & a. 245 & \\
\hline
\end{tabular}




\begin{tabular}{|c|c|c|c|}
\hline $\begin{array}{l}\text { 14) } A E 2001,634 \\
\text { a-c, r. } 7\end{array}$ & [- - ] Heraclida & [aed. II?] & a. 246 \\
\hline $\begin{array}{l}\text { 15) } A E 2001,634 \\
\text { a-c, r. } 10\end{array}$ & {$[---]+e n(--)+++N O[---]$} & [aed. I?] & a. 247 \\
\hline $\begin{array}{l}\text { 16) } A E 2001,634 \\
\text { d, r. } 1\end{array}$ & {$[---]$} & $a[e d . I]$ & $\begin{array}{l}\text { III sec., } \\
\text { metà }\end{array}$ \\
\hline $\begin{array}{l}\text { 17) } A E 2001,634 \\
\text { d, r. } 3\end{array}$ & M. [- - - ] & aed. II & $\begin{array}{l}\text { III sec., } \\
\text { metà }\end{array}$ \\
\hline $\begin{array}{l}\text { 18) Licordari 1984, } \\
\text { p. } 347-349, \text { r. } 1\end{array}$ & A. $E+[---]$ & aed. $p r$. & a. 273 \\
\hline $\begin{array}{l}\text { 19) Licordari } 1984 \text {, } \\
\text { p. } 347-349 \text {, r. } 2\end{array}$ & A. $E[---]$ & aed. sec. & a. 273 \\
\hline $\begin{array}{l}\text { 20) Licordari } 1984 \text {, } \\
\text { p. } 347-349 \text {, r. } 5\end{array}$ & M. $+[---] s$ & aed. $p r$. & a. 274 \\
\hline $\begin{array}{l}\text { 21) Licordari } 1984 \text {, } \\
\text { p. } 347-349 \text {, r. } 9\end{array}$ & 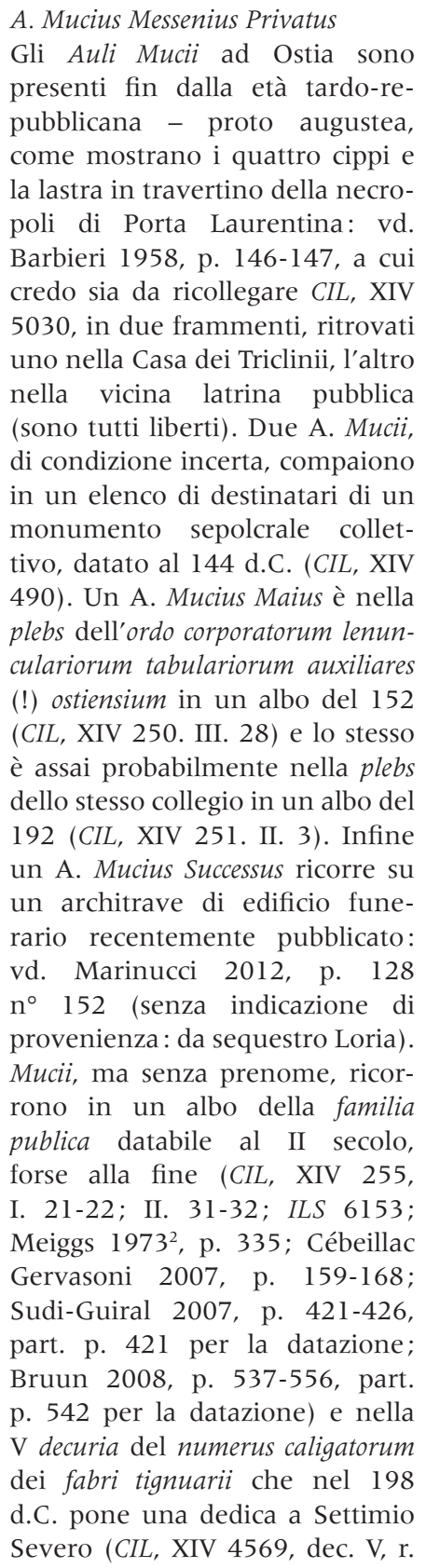 & aed. $p r$. & a. 275 \\
\hline
\end{tabular}




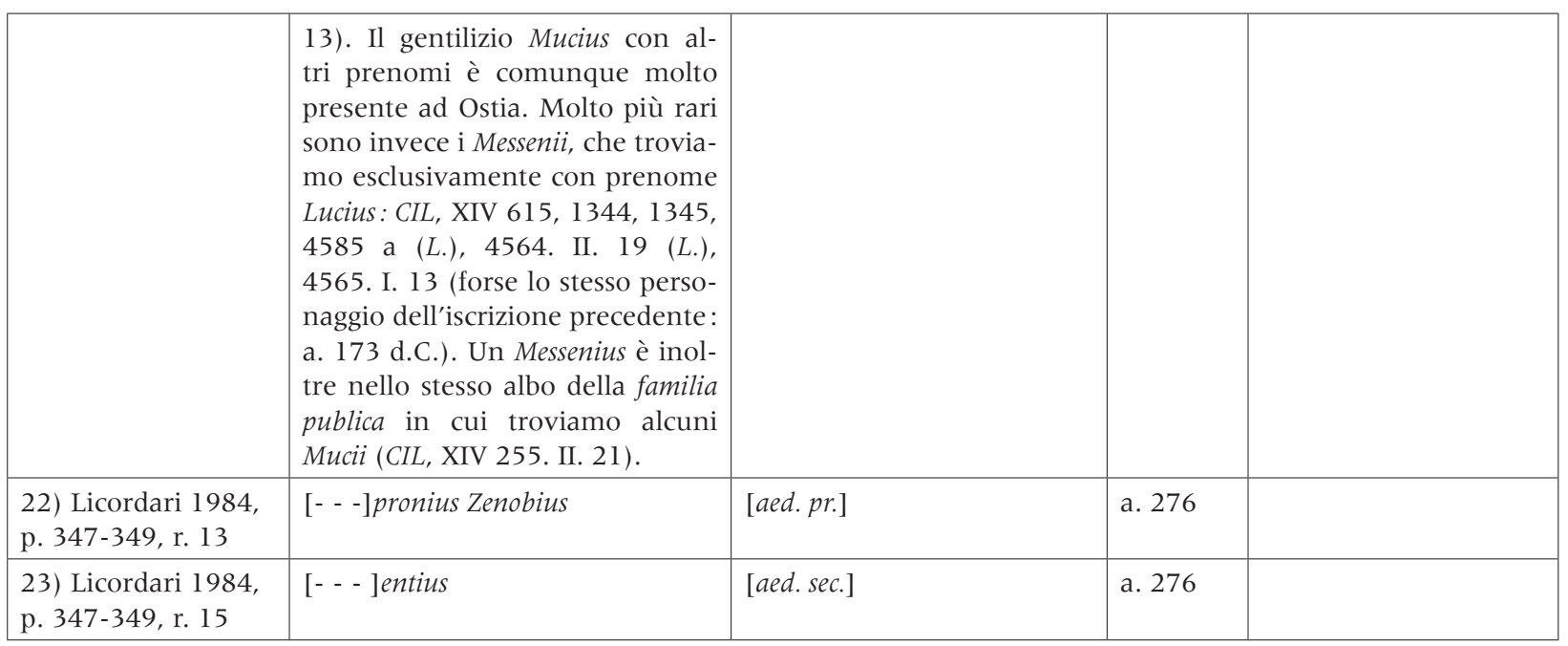

\begin{tabular}{|c|c|c|c|c|}
\hline \multicolumn{5}{|l|}{ PRAETORES } \\
\hline 1) $C I L$, XIV 349 & $\begin{array}{l}\text { C. Fabius Vot. Agrippa } \\
\text { Meiggs 19732, p. } 178 \text { s., 192-193, } \\
\text { 195, 199, 512; Bocherens - Zevi } \\
\text { 2007, p. 257-271, part. } \\
\text { p. 264-265; Zevi 2010, p. 120, } \\
\text { 187. Meiggs poneva il perso- } \\
\text { naggio in età giulio-claudia; ad } \\
\text { una datazione più tarda, pur } \\
\text { nell'ambito del I secolo, pensa } \\
\text { invece Zevi (vd. articolo in questa } \\
\text { stessa rivista). }\end{array}$ & praetor sacris Volkani fac. & I sec. & decurio, aedilis, IIvir \\
\hline 2) $A E 1986,112$ & $\begin{array}{l}\text { A. Caedicius A.f. Saecularis } \\
\text { Il gentilizio Caedicius non è tra i } \\
\text { più comuni ad Ostia. Risulta atte- } \\
\text { stato in CIL, XIV 1373, } 4564 \text {. II. } \\
12 \text { e } 18 \text { (A.), 4565. I. } 12 \text { (è inte- } \\
\text { grato), II, I1 (A.); 4569. XI. } 5 \\
\text { (Caed.); AE 1985, 175; AE 1987, } \\
191 \text { (già FA, 8, 1956, nr. 3680), } \\
\text { dove compare un A. Caedicius } \\
\text { Successus, curator nauclerorum } \\
\text { maris Hadriatici idem q(uin) q(uen- } \\
\text { nalis), cfr. CIL, VI 13876: su } \\
\text { questo vd. Meiggs 19732, p. 276. } \\
\text { Ora ripreso da Marinucci 2012, } \\
\text { p. } 34-35 \mathrm{n}^{\circ} 30 .\end{array}$ & pr. sacris Volk. fac. & I sec. & morto a 5 anni \\
\hline $\begin{array}{l}\text { 3) } C I L, \mathrm{XIV} 412= \\
\text { ILS } 6142\end{array}$ & $\begin{array}{l}\text { Cn. Sergius Cn.f. Vot. Priscus } \\
\text { Meiggs 19732, p. 183, 191, 192, } \\
\text { 205. Cébeillac-Gervasoni 2010, } \\
\text { p. } 195 \text { n }^{\circ} 52 \text {. }\end{array}$ & praetor sacris Volcani & $\begin{array}{l}\text { fine I - } \\
\text { inizio II }\end{array}$ & $\begin{array}{l}\text { figlio di liberto; ex d.d. } \\
\text { aedilis adlectus }\end{array}$ \\
\hline 4) $C I L, X I V 415$ & $\begin{array}{l}\text { C. Silius C.f. Vot. Nerva } \\
\text { Meiggs } 1973^{2} \text {, p. 192, 204. Il genti- } \\
\text { lizio Silius ad Ostia ricorre in CIL, } \\
\text { XIV 245-247, 281, 339,416-417, } \\
1112,1746,1808,4673,5110 \text {; } \\
\text { IPO A 229-231; AE 1995, } 247 . \\
\text { All'iscrizione, che ricorda anche } \\
\text { il padre C. Silius Felix, accennano } \\
\text { Cébeillac-Gervasoni - Zevi 2010, } \\
\text { p. } 166 .\end{array}$ & pr. sacr. vol. fac. & $\begin{array}{l}\text { fine I - } \\
\text { inizio II }\end{array}$ & $\begin{array}{l}\text { figlio di liberto; decu- } \\
\text { rio, Ilvir }\end{array}$ \\
\hline
\end{tabular}




\begin{tabular}{|c|c|c|c|c|}
\hline 5) $A E 1996,304$ & $\begin{array}{l}\text { A. Egrilius A.f. Pal. Magnus } \\
\text { Cfr. CIL, XIV 4899, un'ara fune- } \\
\text { raria in marmo, posta ad un } \\
\text { A. Egrilius A. f. Pal. Magnus, } \\
\text { vissuto } 5 \text { anni. }\end{array}$ & $\begin{array}{l}\text { dec. decr. praet. primus sacris } \\
\text { Volk. faciundis }\end{array}$ & $\begin{array}{l}\text { II sec., } \\
\text { prima } \\
\text { metà }\end{array}$ & $\begin{array}{l}\text { figlio di liberto; morto } \\
\text { a } 8 \text { anni }\end{array}$ \\
\hline $\begin{array}{l}\text { 6) } C I L, \text { XIV } 3=I L S \\
3299\end{array}$ & *Cn. Turpilius Cn.f. Turpilianus & aedil. et pr. sac. Volk. fac. & $\begin{array}{l}\text { II sec., } \\
\text { prima } \\
\text { metà }\end{array}$ & $\begin{array}{l}\text { non ricopre altre } \\
\text { cariche }\end{array}$ \\
\hline $\begin{array}{l}\text { 7) } A E 1988,202= \\
\text { Bloch 1953, } \mathrm{n}^{\circ} 50\end{array}$ & P. Attius P.f. Pal. Silianus & praet. sacri. Volk. faciundis & $\begin{array}{l}\text { II, prima } \\
\text { metà }\end{array}$ & $\begin{array}{l}\text { figlio di liberto; non } \\
\text { ricopre altre cariche }\end{array}$ \\
\hline $\begin{array}{l}\text { 8) } C I L, \text { XIV } 306= \\
\text { ILS } 6143\end{array}$ & $\begin{array}{l}\text { L. Aurelius L.f. Pal. Fortunatianus } \\
\text { Meiggs } 1973^{2} \text {, p. } 338 \text {. Cfr. il L. } \\
\text { Aurelius Fortunatus noto dall'i- } \\
\text { scrizione ostiense edita da Calza } \\
\text { 1939, p. } 28 \text { (non anteriore al } 154 \\
\text { d.C.). }\end{array}$ & pr. pr. sacr. Volka. & $\begin{array}{l}\text { II sec., } \\
\text { metà }\end{array}$ & morto a 4 anni \\
\hline 9) CIL, XIV 376 & *P. Lucilius Gamala Iunior & $\begin{array}{l}\text { aed. sacr. Volcani, eiusdem pr. } \\
\text { tert. (poi pontifex) }\end{array}$ & $\begin{array}{l}\text { II sec., } \\
\text { seconda } \\
\text { metà }\end{array}$ & $\begin{array}{l}\text { decurio, IIvir praefectus } \\
\text { L. Caesar(is) Aug(usti) } \\
\text { f(ili) cens(or), quaes- } \\
\text { tor aerarii, tabular. et } \\
\text { librorum curator, curator } \\
\text { pecuniae publicae exi- } \\
\text { gendae et attribuendae }\end{array}$ \\
\hline $\begin{array}{l}\text { 10) } C I L, \text { XIV } 341= \\
\text { ILS } 6144\end{array}$ & 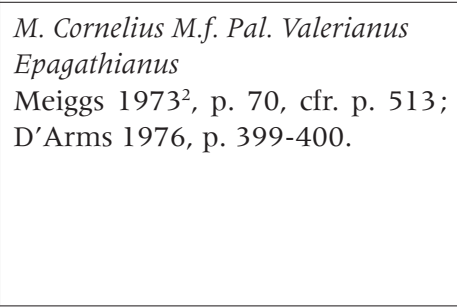 & praetor II sacra Volkani fac. & $\begin{array}{l}\text { II sec., } \\
\text { fine }\end{array}$ & $\begin{array}{l}\text { cavaliere, decu- } \\
\text { rio, flamen, sodalis } \\
\text { arulensis, decurio et } \\
\text { IIIIvir Laurentium vici } \\
\text { Aug., patronus corporis } \\
\text { lenunculariorum tabu- } \\
\text { lariorum auxiliariorum } \\
\text { Ostiensium }\end{array}$ \\
\hline $\begin{array}{l}\text { 11) } C I L, \text { XIV } 4625= \\
\text { Bloch } 1953, \mathrm{n}^{\circ} 56, \\
\text { cfr. } A E 1988,207\end{array}$ & $\begin{array}{l}\text { D. Iunius D.f. Pal. Bubalus } \\
\text { Impetratus } \\
\text { Meiggs } 1973^{2} \text {, p. } 211 \text {, cfr. p. } 513 \text {; } \\
\text { D'Arms } 1976, \text { p. } 401-402 .\end{array}$ & $\begin{array}{l}\text { praetor primus sacris Volkani } \\
\text { faciund. }\end{array}$ & $\begin{array}{l}\text { II sec., } \\
\text { fine }\end{array}$ & $\begin{array}{l}\text { cavaliere, decu- } \\
\text { rio a Ostia, decurio } \\
\text { Laurentium vicanorum, } \\
\text { Arulensis }\end{array}$ \\
\hline $\begin{array}{l}\text { 12) CIL, XIV } 4138 \text {, } \\
\text { r. } 1\end{array}$ & $+[---]$ & pr. I & a. 192 & \\
\hline $\begin{array}{l}\text { 13) CIL, XIV } 4138 \text {, } \\
\text { r. } 3\end{array}$ & {$[---]$} & pr.II & a. 192 & \\
\hline $\begin{array}{l}\text { 14) } C I L, \text { XIV } 4138 \text {, } \\
\text { r. } 7\end{array}$ & {$[---]$} & $p[r . I]$ & a. 193 & \\
\hline $\begin{array}{l}\text { 15) CIL, XIV } 373= \\
\text { ILS } 6141\end{array}$ & $\begin{array}{l}\text { L. Licinius L.fil. Pal. Herodes } \\
\text { Meiggs 19732, p. } 210 ; \text { D'Arms }^{2} \\
\text { 1976, p. } 393 .\end{array}$ & praet. prim. sac. Volk. faciu. & $\begin{array}{l}\text { post. } 211 \\
\text { d.C. }\end{array}$ & $\begin{array}{l}\text { cavaliere, decurialis de- } \\
\text { curiae viatoriae equestris } \\
\text { cos., decurio, quinquen- } \\
\text { nalis duumvir, sacerdos } \\
\text { geni col., flam. divi } \\
\text { Severi, sodalis Arulensis }\end{array}$ \\
\hline $\begin{array}{l}\text { 16) } A E, 1995,244 \text {, } \\
\text { r. } 1\end{array}$ & $\begin{array}{l}\text { Q. Flo[- - - } \\
\text { Forse Flo[rius] in base alla } \\
\text { frequenza delle attestazioni ad } \\
\text { Ostia. E' tuttavia da ricordare che, } \\
\text { in relazione a questo gentilizio, } \\
\text { ricorre solo il prenome Lucius. Vd. } \\
\text { Salomies 2002, p. } 157 \text {. }\end{array}$ & pr. II & $\begin{array}{l}\text { a. } 221 \\
\text { o } 225 \text { o } \\
228\end{array}$ & \\
\hline
\end{tabular}




\begin{tabular}{|c|c|c|c|c|}
\hline $\begin{array}{l}\text { 17) } A E, 1995,244 \text {, } \\
\text { r. } 4\end{array}$ & $\begin{array}{l}\text { M. Tut[- - - } \\
\text { Forse Tut[ilius] in base alla fre- } \\
\text { quenza delle attestazioni ad Ostia. } \\
\text { E' tuttavia da ricordare che, in rela- } \\
\text { zione a questo gentilizio, i prenomi } \\
\text { ricorrenti sono Caius, Cnaeus e } \\
\text { Lucius. Vd. Salomies 2002, p. } 157 \text {. }\end{array}$ & pr. I & $\begin{array}{l}\text { a. } 222 \\
\text { o } 226 \text { o } \\
229\end{array}$ & \\
\hline $\begin{array}{l}\text { 18) } A E, 1995,244 \text {, } \\
\text { r. } 6\end{array}$ & $+[---]$ & pr. II & $\begin{array}{l}\text { a. } 222 \\
\text { o } 226 \text { o } \\
229\end{array}$ & \\
\hline $\begin{array}{l}\text { 19) } A E, 2001,634 \\
\text { a-c, r. } 2\end{array}$ & $\begin{array}{l}\text { Q. Ve[- - Ae]milius Pacatus } \\
\text { Possibili le integrazioni dei } \\
\text { gentilizi Vergilius (sempre con } \\
\text { prenome Quintus), Vettius e Vetu- } \\
\text { rius (entrambi anche con prenome } \\
\text { Quintus). Un Q. Veturius è pretore } \\
\text { di Vulcano intorno al } 251 \text { d.C. Un } \\
\text { Q. Vettius è pontefice sullo scorcio } \\
\text { del III secolo. Il gentilizio poteva } \\
\text { essere per esteso o abbreviato, } \\
\text { come altrimenti attestato nel fram- } \\
\text { mento degli anni } 245-247 \text { d.C. }\end{array}$ & {$[p] r . p r$.} & a. 245 & \\
\hline $\begin{array}{l}\text { 20) } A E, 2001,634 \\
\text { a-c, r. } 3\end{array}$ & M. Aur $[--$ Ca]ndidian $[u s]$ & {$[p] r . I I$} & a. 245 & \\
\hline $\begin{array}{l}\text { 21) } A E, 2001,634 \\
\text { a-c, r. } 6\end{array}$ & [- - ] Atticus & [pr. I] & a. 246 & \\
\hline $\begin{array}{l}\text { 22) } A E, 2001,634 \\
\text { a-c, r. } 9\end{array}$ & [- - ]en(- - ) Privatus & [pr. I] & a. 247 & \\
\hline $\begin{array}{l}\text { 23) Nuzzo } 1999 \text {, } \\
\text { p. } 40 \mathrm{n}^{\circ} \mathrm{A} 6\end{array}$ & {$[---]$} & pr. II & $\begin{array}{l}\text { III sec., } \\
\text { metà }\end{array}$ & \\
\hline $\begin{array}{l}\text { 24) } A E, 2001,634 \\
\text { d, r. } 2\end{array}$ & {$[---]$} & pr. II & $\begin{array}{l}\text { III sec., } \\
\text { metà }\end{array}$ & \\
\hline $\begin{array}{l}\text { 25) } A E, 2001,634 \\
\text { e, r. } 2\end{array}$ & {$[---]$} & pr. I & $\begin{array}{l}\text { III sec., } \\
\text { metà }\end{array}$ & \\
\hline 26) $C I L$, XIV 432 & $\begin{array}{l}{[Q . \text { Vetu }] \text { rius Firmius }[\text { Felix }]} \\
\text { Socrates } \\
\text { Meiggs } 1973^{2}, \text { p. } 210 ; \text { D'Arms }^{\prime} \\
\text { 1976, p. } 395-396 .\end{array}$ & $\begin{array}{l}\text { [praet.? pr] imus sac. [Volk. } \\
\text { fa]ciundis }\end{array}$ & $\begin{array}{l}\text { ca. } 251 \\
\text { d.C. }\end{array}$ & $\begin{array}{l}q(\text { uin) } q \text { (uennalis) } c(\text { en- } \\
\text { soria) } p \text { (otestate) (cfr. } \\
\text { CIL, XIV 352b), decurio }\end{array}$ \\
\hline $\begin{array}{l}\text { 27) Licordari } 1984 \text {, } \\
\text { p. } 347-349, \text { r. } 4\end{array}$ & C. $+[---]$ & pr.pr. & a. 273 & \\
\hline $\begin{array}{l}\text { 28) Licordari } 1984 \text {, } \\
\text { p. } 347-349 \text {, r. } 6\end{array}$ & $\begin{array}{l}\text { A. [.]d[- - - ]s Furius }[---] s \\
\text { Furius è gentilizio molto diffuso } \\
\text { ad Ostia. }\end{array}$ & pr. sec. & a. 274 & \\
\hline $\begin{array}{l}\text { 29) Licordari } 1984 \text {, } \\
\text { p. } 347-349, \text { r. } 8\end{array}$ & M. Aurelius Pegasius & pr.pr. & a. 275 & \\
\hline $\begin{array}{l}\text { 30) Licordari } 1984 \text {, } \\
\text { p. } 347-349 \text {, r. } 10\end{array}$ & $\begin{array}{l}\text { A. Mucius Messenius Marcellinus } \\
\text { Sui Mucii e i Messenii vd. supra } \\
\text { nota } 45 .\end{array}$ & pr. sec. & a. 275 & \\
\hline $\begin{array}{l}\text { 31) Licordari } 1984 \text {, } \\
\text { p. } 347-349, \text { r. } 12\end{array}$ & [-- -]cius Menedemus & [pr.pr.] & a. 276 & \\
\hline $\begin{array}{l}\text { 32) Licordari } 1984 \text {, } \\
\text { p. } 347-349, \text { r. } 14\end{array}$ & [-- -] iun. & [pr. sec.] & a. 276 & \\
\hline 33) CIL, XIV 402 & $\begin{array}{l}{[---] \text { Poll }[---]} \\
\text { Si tratta di un frammento di } \\
\text { lastra marmorea, } 27 \times 27 \times 3 . \mathrm{Si} \\
\text { conserva ad Ostia, deposito } 20 \text {, } \\
\text { scaffale 52C, inv. } 8004 \text {. }\end{array}$ & pr. sacr. [Volk. fac.] & $\begin{array}{l}\text { indata- } \\
\text { bile }\end{array}$ & \\
\hline
\end{tabular}




\begin{tabular}{|c|c|c|c|c|}
\hline \multicolumn{5}{|c|}{ AEDILES VEL PRAETORES } \\
\hline$A E 1986,111$ & $\begin{array}{l}\text { [Ti.? Clau?]dius Civi[lis?] } \\
\text { Vd. ora Marinucci 2012, p. } 79 \\
\text { n } 98 .\end{array}$ & [-- sa]c. vol. fac. & I sec. & \\
\hline $\begin{array}{l}\text { CIL, XIV 4648, rr. } \\
2-6\end{array}$ & [A. Egrilius?] Faustinus & {$[---] f a c\ulcorner i \neg u n d$.} & $\begin{array}{l}\text { fine II - } \\
\text { inizio III }\end{array}$ & $\begin{array}{l}\text { cavaliere, flamen divi } \\
\text { Pertinacis, aedilis, quaes- } \\
\text { tor aerarii }\end{array}$ \\
\hline $\begin{array}{l}\text { CIL, XIV 4648, rr. } \\
\text { 7-8 }\end{array}$ & A. Egrilius $[---]$ & {$[---$ V]olc. $[---]$} & $\begin{array}{l}\text { fine II - } \\
\text { inizio III }\end{array}$ & \\
\hline
\end{tabular}

La prima, isolata, attestazione degli aediles sacris Volkani faciundis è dell'ultimo secolo della Repubblica: si tratta di P. Lucilius Gamala Senior, che, oltre a ricoprire le magistrature cittadine, fu poi anche pontifex del culto. L'unico altro caso noto è quello del suo lontano discendente $P$. Lucilius Gamala Iunior (vd. infra). Dei successivi personaggi nove si concentrano nel II secolo. Di questi, quattro non ricoprirono altre cariche: nel caso di A. Fabius Felicianus e di $N$. Trebonius Venerius la ragione sarà da ricercarsi certamente nella morte precoce, rispettivamente a diciannove e forse non più di tredici anni. L'ultimo era con certezza figlio di un liberto.

Dei restanti cinque, uno è il noto $P$. Lucilius Gamala Iunior, discendente di una delle famiglie radicate nella élite locale dall'ultimo secolo della Repubblica. Del tutto particolare è la sua posizione nel culto: prima aedilis, poi praetor tertius, infine pontifex, in modo analogo ma non identico al suo antenato.

Diversa è invece l'estrazione sociale dei due edili A. Egrilius Paternus e P. Nonius Livius Anterotianus. Il primo è figlio di due probabili liberti: Egrilia Daphne e A. Egrilius Onesimus, coactor, sevir Augustalis idem q(uin) q(uennalis). Tale condizione non gli ha impedito l'accesso all'ordine equestre ${ }^{35}$, nel quale dovette entrare a partire dalla sua precedente carriera apparitoria (era lictor curiatius $^{36}$ ). Ad Ostia fu anche flamen divi Vespasiani. Il secondo era invece nipote di un liberto, A. Livius Anteros, e venne poi adottato da un P. Nonius. Anche lui entrato nell'ordine equestre ${ }^{37}$, ad Ostia fu decurione, flamen divi Hadriani e salius Laurentium Lavinatium.

35. Demougin 1994, p. 370-375 (cit. alle p. 374 e 375). Sulla questione dei liberti entrati nell'ordine equestre vd. Eck 1999, p. 14; Demougin 1992, p. 107-121, part. p. 111-116; López Barja de Quiroga 1995, p. 326-348.

36. Vd. Scheid 1999, p. 142. Caso analogo è quello del liberto L. Marius L. lib. Doryphorus, noto da CIL, VI $1847=I L S 1899$.

37. Demougin 1994, p. 370-375 (cit. alle p. 374 e 375).
Dei due edili del 192 nulla possiamo dire, dato lo stato della documentazione. Stesso discorso riguarda i tredici edili che i Fasti ci fanno conoscere per il III secolo, tranne in parte per A. Mucius Messenius Privatus, alle spalle del quale sembra di poter distinguere una origine libertina della famiglia più o meno lontana.

Le attestazioni dei praetores sacris Volkani faciundis, assenti (ma forse solo fortuitamente) per l'età repubblicana, si spalmano, sia pur non equamente, sui primi tre secoli dell'Impero. Dei trentatre personaggi noti, cinque non sembrano aver ricoperto altre cariche (per altri non si può dire, data l'estrema lacunosità dei documenti): come per gli aediles, nel caso di A. Caedicius Saecularis, A. Egrilius Magnus e L. Aurelius Fortunatianus la ragione deve essere ascritta alla morte precoce, sopraggiunta rispettivamente a cinque, otto e quattro anni. Ragione analoga può riguardare P. Attius Silianus, dal momento che l'iscrizione sepolcrale venne posta dal padre. Più difficile è invece pronunciarsi nel caso di Cn. Turpilius Turpilianus, noto da una dedica sacra, che lo ricorda aedilis e praetor del culto. A. Egrilius Magnus e P. Attius Silianus erano entrambi figli di liberti: il padre del primo, A. Aegrilius (!) Heliades, e quello del secondo, P. Attius Felicio, furono ad Ostia seviri Augustales idem quinquennales.

Per altri otto casi, in cui abbiamo la possibilità di seguire le vicende del personaggio, la situazione è più complessa. Una linea di demarcazione sembra potersi tracciare alla metà del II secolo. 
Prima di tale data, oltre ai cinque personaggi ricordati sopra, abbiamo a che fare con tre individui che, a prescindere dalla loro origine, non sembrano uscire dalla élite locale. C. Fabius Agrippa, figlio e nipote di primipili radicati nella colonia, fu ad Ostia decurio, aedilis e IIvir; Cn. Sergius Priscus, figlio di un liberto ed Augustalis, venne cooptato edile, se è corretta questa lettura del testo ${ }^{38}$; C. Silius Nerva, anche lui figlio di liberto ed Augustalis ${ }^{39}$, fu, al pari del primo, decurio, aedilis e IIvir.

Figura di passaggio tra questo tipo di praetores e quelli attestati dall'ultimo quarto del II secolo è P. Lucilius Gamala Iunior: rampollo, come si è visto, di una famiglia che da due secoli forniva magistrati alla colonia, fu aedilis, poi praetor tertius, infine pontifex del culto di Vulcano e al contempo decurio, IIvir praefectus L. Caesar(is) Aug(usti) f(ili), ossia di Commodo, nel 171 o 176, cens(or), quaestor aerarii, tabular(um) et librorum curator, curator pecuniae publicae exigendae et attribuendae. Un uomo di spicco della colonia, pur senza varcarne i limiti, e comunque con solidi legami presso l'amministrazione centrale, come mostra la prefettura locale in luogo del giovane Commodo.

Con M. Cornelius M.f. Pal. Valerianus Epagathianus, D. Iunius D.f. Pal. Bubalus Impetratus e L. Licinius L.fil. Pal. Herodes la situazione cambia. Senza poter escludere per nessuno di loro una più o meno lontana origine libertina, ben diversa è la loro ascesa sociale.

Il primo, M. Cornelius M.f. Pal. Valerianus Epagathianus, era cavaliere romano ${ }^{40}$; a Ostia fu decurione, flamen di un culto non specificato, praetor secundus di Vulcano, sodalis Arulensis, patrono del corpus dei lenuncularii tabularii auxi-

38. È in realtà possibile anche la lettura aedilicius. Sul termine vd. ora Salomies 2010, p. 213-215, 225-229 (per considerazioni di ordine cronologico: l'uso sembra diffondersi dal tardo II secolo fino al IV). L'iscrizione in esame non è presa in considerazione. Sembra da riferire a questa nomina l'espressione ex d(ecurionum) d(ecreto).

39. Il padre di Nerva, C. Silius Felix maior, aveva ricevuto dai decurioni l'onore di un funus publicum: per questo vd. Gregori 2007, p. 1074; Caldelli 2008, p. 264-265.

40. L'iscrizione è nota da tradizione manoscritta e, per quanto riguarda la durata della vita, tutti tramandano XII, tranne il Gruter (non accolto da Dessau) che indica XLI. È vero che l'iscrizione è posta dal padre, come sembrerebbe per ragioni onomastiche e per la presenza della sigla $f$. da sciogliersi con f(ilio), ma il cumulo di cariche rivestite mi sembra difficilmente compatibile con una morte a 12 anni. liarii; nel vicino Vicus Augustanorum fu decurione e, forse, quattuorviro ${ }^{41}$.

Il secondo, D. Iunius D.f. Pal. Bubalus Impetratus, ebbe una carriera in tutto simile al precedente: anche lui cavaliere romano, a Ostia fu decurione, praetor primus di Vulcano e sodalis Arulensis; fu decurione pure nel vicino Vicus Augustanorum ${ }^{42}$.

Il terzo, infine, L. Licinius L.fil. Pal. Herodes, condivide con i precedenti la condizione di cavaliere romano e, ad Ostia, la carica di decurione ed i sacerdozi di praetor primus di Vulcano e di sodalis Arulensis; a questo bisogna però aggiungere, ad Ostia, l'intero cursus locale (qui fu, infatti, quaestor aerari, aedilis e duovir quinquennalis) comprensivo anche della curatela delle opere pubbliche, del sacerdozio del Genius coloniae, del flaminato di Roma e di Augusto e del flaminato del divus Severus; a Roma, invece, prima di essere ammesso nell'ordine equestre, fu membro di una decuria viatoria equestris dei consoli ${ }^{43}$.

Tutti e tre indicano di appartenere alla tribù Palatina e, anche se oggi si tende a sfumare l'implicazione che ciò comporti una più o meno lontana origine libertina ${ }^{44}$, una appartenenza familiare, forse non recentissima, al gruppo dei liberti va ipotizzata nel primo e nel terzo caso: nel primo per la fitta presenza di liberti con stesso prenome e gentilizio negli albi collegiali, nel terzo in ragione del dedicante stesso della base onoraria posta ad Herodes, vale a dire l'ordo Augustalium.

Origine libertina o no, questo poco importa in un'epoca - fine del II-inizio III secolo d.C. - in cui la classe dirigente ostiense è comunque per lo più formata o da discendenti di liberti o da immigrati. Quello che invece colpisce è l'impennata sociale, come se il vecchio culto di Vulcano, almeno ai suoi livelli più bassi, venisse affidato a famiglie che potenzialmente potessero non solo gestire la cosa pubblica ad Ostia ma anche aspirare agli ordini superiori.

Se si getta uno sguardo al pontificato di Vulcano, la situazione appare in parte differente: nella lista dei pontefici apparentemente l'affidamento della carica a personaggi dell'élite locale entrati nella amministrazione centrale sembra

\footnotetext{
41. Vd. supra nota 52.

42. Vd. supra nota 53.

43. Vd. supra nota 54.

44. Cébeillac-Gervasoni - Zevi 2010, p. 161-169.
} 
iniziare dai primi anni del II secolo con M. Acilius Priscus Egrilius Plarianus. Gli Egrilii, ormai senatori, sembrano monopolizzare il pontificato negli anni di Traiano e di Adriano, anni importanti per la colonia anche sotto il profilo religioso.

La sequenza della seconda metà del II secolo è molto lacunosa. Due i punti fermi: P. Lucilius Gamala Iunior e M. Antius Crescens Calpurnianus. Nel terzo quarto del secolo va collocata la figura di P. Lucilius Gamala Iunior, come si è visto aedilis, poi praetor tertius, infine pontifex: a fronte delle sua brillante carriera locale e dei suoi natali, non riuscì ad entrare negli ordini superiori. Alla fine del secolo si colloca M. Antius Crescens Calpurnianus, che due iscrizioni ostiensi, datate rispettivamente al 194 e al 203, fanno conoscere come pontefice di Vulcano ${ }^{45}$ : vir clarissimus, fu pretore, curator rei publicae Mars[orum Marruviorum], legatus pro praetore di una provincia di cui è andato perduto il nome, iuridicus Britanniae vice legati, proconsole in Macedonia, console suffetto in anno ignoto da collocare all'inizio del III secolo; XVvir sacris faciundis e, forse come tale, menzionato negli Acta dei ludi saeculares del $204^{46}$.

Più complessa è nella sequenza la posizione di C. Nasennius Marcellus. Nella serie degli omonimi credo si possa considerare acquisita l'identità tra C. Nasennius Marcellus Senior, che compare in CIL, XIV 171 e nella lacunosa CIL, XIV 4457 con colui che ricoprì per la terza volta il duovirato in anno di censo nel 111 d.C., quale attestato dai Fasti citta$\operatorname{dini}^{47}$. Più complessa è la distribuzione dei restanti documenti. Al contrario di Meiggs ${ }^{48}$, che non considera due documenti solo di recente ricondotti ad Ostia, preferirei distinguere altre due generazioni successive (non una sola), costituite una dal C. Nasennius Marcellus, noto da CIL, XIV 4148 come IIvir quinquennalisnel 166 d.C. e un'altra dal C. Nasennius Marcellus, IIvir nel $180^{49}$, curator operum

45. CIL, XIV 325, cfr. p. $615=$ CCCA, III, p. 110 s. n $363=$ Rieger 2004, p. $294 n^{\circ}$ MM58 fig. 121 a-b (a. 194 d.C.) e CIL, XIV 324 , cfr. p. $614=I L S 4176=$ CCCA, III, p. 111 s. n $364=$ Rieger 2004, p. $294 \mathrm{n}^{\circ}$ MM59 fig. 120 a-b (a. 203 d.C.).

46. Il cursus del personaggio si ricostruisce da CIL, VI 41177 (già 1136, cfr. 31634). Il suo nome compare negli Atti dei Ludi saeculares del 204 d.C.: CIL, VI 32326, 50; 32327, 10 ; 32332, 3. Vd. PIR2, A 781; Salomies 1996, p. 71.

47. Vidman 19822, fram. J e p. 105.

48. Meiggs 19732, p. 509-510.

49. Marinucci 1988, p. $200 \mathrm{n}^{\circ} 26$ : è vero che il nome è parzialmente integrato [C.] Nasenni[o Marcello?]. publicorum nel $181^{50}$, curator $p$ (er)p(etuus) operum publicorum (come il nonno) nel $184^{51}$ e patronus coloniae nel $189^{52}$. Penso che questo vada identificato con il pontifex Volcani et aedium sacrarum di CIL, XIV $47^{53}$ e, tornando alla posizione di Grosso in merito ${ }^{54}$, penso che nella sequenza dei pontefici vada piuttosto inserito tra Gamala Iunior e Crescens Calpurnianus e non posposto ad esso. La esatta condizione sociale degli ultimi due Nasenni Marcelli e in particolare del pontefice ci sfugge. Il nonno, che pure era stato curator $p$ (er) $p$ (etuus) operum publicorum, era entrato nell'ordine equestre e aveva ricoperto alcune cariche di debutto.

Ignota è anche la esatta condizione sociale dei più tardi pontefici del culto, Iulius Faustinus, attestato da un'iscrizione datata al 251 d.C. ${ }^{55}$ e $Q$. Vettius Postumius Constantius, vissuto sullo scorcio del III secolo ${ }^{56}$.

Pur con tutte le lacune della documentazione, per quanto riguarda il pontificato di Vulcano sembra di poter dire che questo sia cresciuto d'importanza (anche agli occhi di Roma) dall'inizio del II secolo, cioè dalla costruzione del porto di Traiano, tanto che da allora dovesse essere di preferenza rivestito solo da ostiensi entrati negli ordini superiori. La sola eccezione certa, quella di Gamala Iunior, si può spiegare da una parte con l'impossibilità di trovare sempre ostiensi "titolati », dall'altra con l'indubbio prestigio, almeno ad un livello locale allargato, della famiglia.

Per contro, per quanto riguarda i sacerdoti minori di Vulcano, viene da chiedersi se non esista una fase commodiana. L'interesse di Commodo per Ostia è stato già in passato sottolineato ${ }^{57}$ : forse per contrastare gli effetti della grande carestia e delle pestilenze dell'età di Marco Aurelio e Commodo ${ }^{58}$,

50. CIL, VI 861, cfr. p. $3007=C I L, X 1791=I L M N$, I, 563.

51. CIL, XIV $172+$ add. p. 481.

52. CIL, XIV 460.

53. L'iscrizione è stata ripresa da G. Sacco in IGI. Porto, 13, che pure propende per questa identificazione, aggiungendo che l'iscrizione, per paleografia, si inquadra bene nella seconda metà del II secolo d.C.

54. Grosso 1959, p. 292-293.

55. CIL, XIV $352=I L S 6149 ;$ RICIS, II, 503/1115 con bibliografia precedente. Ma vd. ora Caldelli 2014, c.s.

56. Vd. supra nota 14

57. Meiggs 19732, p. 79-80, 549.

58. Sul tema vd. ora la raccolta di studi in Lo Cascio 2012; minimizza gli effetti su Ostia Bruun 2003, p. 426-434, part. p. 433-434. Sulla pestilenza sotto Commodo vd. in particolare Lo Cascio 2002, p. 102-106; Storchi Marino 2012, p. 52-53, 56-58. 
la riorganizzazione del traffico annonario ${ }^{59}$ avrebbe avuto una ricaduta positiva sulla colonia come mostrerebbero, solo per ricordare gli interventi più vistosi, il nuovo impulso alla costruzione o ricostruzione degli horrea ${ }^{60}$ e l'ampliamento ed il restauro del teatro ${ }^{61}$ e della adiacente porticus ${ }^{62}$. Fulvio Grosso, che pure era cauto nei confronti di interpretazioni eccessivamente ottimistiche del consenso degli ostiensi nei confronti della politica di Commodo $^{63}$, non poteva non notare come le due uniche attestazioni del nome Commodus riferito al mese di agosto venissero da Lanuvio, città natale dell'imperatore, e proprio da Ostia, dove, peraltro, compare in un documento non ufficiale ${ }^{64}$.

Ma la fase commodiana è stata valorizzata anche di recente nel quadro dello sviluppo dei collegia legati all'annona e al suo indotto (fabri tignuarii): uno studio di Fausto Zevi ha messo in evidenza un incremento nella visibilità di alcuni collegi, quali quelli dei fabri navales, dei fabri tignuarii, dei pistores, proprio a partire dagli anni '90 del II secolo, espressa attraverso la costruzione delle relative scholae in luoghi centrali della colonia o comunque di grande passaggio e con ostentatorio sfarzo nell'apparato. Se anche la conclusione di molte di queste iniziative risale ai primi anni di regno di Settimio Severo, si può ragionevolmente ipotizzare che siano "l'esito di iniziative avviate

59. Nel quadro della quale è ora da riconsiderare il significato attribuito alla notizia della HA, V. Comm., 17. 7 circa la creazione di una classis Africana, cioè di una flotta apposita che avrebbe dovuto trasportare il grano africano a Roma, al posto di quello egiziano: vd. al riguardo Pavis d'Escurac 1974, p. 397-408. La studiosa pensa si tratti piuttosto di un distaccamento militare posto a difesa delle coste africane. Contra, a sostegno dell'interpretazione corrente Lo Cascio 2002, p. 103.

60. Rickman 1971, p. 41 (horrea Antoniniani), 51 (grandi horrea).

61. CIL, XIV 114: proprio l'ampliamento del teatro viene utilizzato da Bruun 2003, p. 426-434, part. p. 433-434 per minimizzare gli effetti della peste su Ostia. I lavori, conclusi nel 196 d.C., come apprendiamo dall'iscrizione, iniziarono sotto Commodo, come attestano i bolli: su questo vd. Pensabene 2002, p. 268; Battistelli 2002, p. 416 nota 55.

62. Pohl 1978a, p. 333-334; Pohl 1978b, p. 193.

63. Da ridimensionare sarebbe dunque, seguendo la linea interpretativa dello studioso, il significato delle dediche poste a Commodo pro salute: CIL, XIV 22 (e a Iuppiter Dolichenus), 24 (e a Iuppiter Optimus Maximus Helipolitanus), 30 (e a Liber Pater Commodianus), 4301 (taurobolium); 4378.

64. CIL, XIV $2113=$ ILS 5193 (base di statua da Lanuvio: a. 187 d.C.) e CIL, XIV 5291, 2 (graffito da Ostia, casa di Giove e Ganimede, senza datazione consolare): VII kal(endas) Commodas. Vd. Grosso 1964, p. 215-217, cfr. p. 239 nota 2. già prima" e proprio nell'età di Commodo ${ }^{65}$. A sostegno di tale linea interpretativa, lo studioso da una parte sottolinea come la grande disponibilità di spesso prestigiose proprietà immobiliari passate da privati a collegi possa spiegarsi come il portato delle condanne e confische dell'età di Commodo, dall'altra ricorda la grande lastra iscritta, pubblicata alcuni anni fa, in cui si fa menzione della assegnazione di un terreno per la sede del locale corpus pistorum da parte del prefetto dell'annona M. Aurelius Papirius Dionysius, famoso giurista strettamente legato a Commodo e nondimeno messo a morte dal principe probabilmente nel $191^{66}$, attivo ad Ostia nella tarda età commodiana.

Ma l'impronta di Commodo ad Ostia sembra delinearsi anche a livello cultuale. Come è noto, l'imperatore aveva una speciale devozione per Ercole, con il quale intese identificarsi ${ }^{67}$. Ostia, già sede di un radicato culto di Ercole, sembra essere stato l'unico centro, oltre Roma ${ }^{68}$, ad aver ospitato i sodales Herculani ${ }^{69}$, la cui istituzione si può attribuire a Commodo.

Per questa via e alla luce delle testimonianze raccolte l'età di Commodo sembra segnare una svolta anche nel sacerdozio dell'antico culto di Vulcano: se con il pontificato affidato a personaggi degli ordini superiori a partire dall'età traianea il controllo sulle aedes pubbliche è sempre meno un affare della colonia, la apparente riqualificazione dell'edilità e della pretura di Vulcano può essere letta come una espressione della maggiore ingerenza di Roma nel culto, che sembra favorire individui destinati ad uscire dalla élite locale. Figura di passaggio sembra essere P. Lucilius Gamala Iunior,

65. Vd. Zevi 2008, p. 494.

66. Zevi 2008, p. 477-505. L'iscrizione citata è pubblicata da Nuzzo 1996, p. 90-91, con foto, cfr. $A E$ 1996, 309; Nuzzo 1999, p. 38-39 n A4, con foto. Su M. Aurelius Papirius Dionysius vd. Whittaker 1964, p. 348-369; Pavis d'Escurac 1976, p. 352-353.

67. Grosso 1964, p. 333-344; Hekster 2002, p. 11-13, 103-111, 117-120.

68. CIL, VI 1339, cfr. cfr. p. 3141, 3805, 4683 = ILS 1121 e CIL, VI 31691, cfr. p. 3805, $4775=$ ILS 1120 .

69. Bloch 1953, p. 288-289 n 49, cfr. $A E$ 1988, 201 (la lettura della r. 1 va corretta in Pal(atina)) e Bloch 1953, p. 291-292, $\mathrm{n}^{\circ}$ 54, cfr. $A E$ 1982, 132 e vd. anche $A E$ 1991, 333. Vd. Meiggs 19732, p. 79, 185. Sul significato di queste iscrizioni torna Mar 1996, p. 148-164: l'A., tuttavia, non specifica l'esatta fase cronologica di appartenenza della documentazione epigrafica. Sulla questione dell'ingerenza di Roma nella vita cultuale della colonia vd. van Haeperen 2006, p. 31-50. 
con la sua anomala carriera che lo vede prima aedilis, poi praetor, addirittura tertius, infine pontifex, e che, in qualità di praefectus, sostituisce proprio il giovane Commodo, eletto duoviro. Se l'ipotesi cogliesse nel vero, a questo punto saremmo autorizzati a supporre che anche la redazione dei Fasti dei sacerdoti minori di Vulcano sia una novità dell'ultimo quarto del II secolo.

\section{Bibliografia}

Balbi de Caro 1968 = S. Balbi de Caro, Un nuovo pontefice di Vulcano ad Ostia, in Epigraphica, 30, 1968, p. 75-82.

Battistelli 2002 = P. Battistelli, Lo sviluppo architettonico del complesso del teatro di Ostia alla luce delle recenti indagini nell'edificio scenico, in MEFRA, 114, 1, 2002, p. 391-420 (con G. Greco).

Baurain $1976=\mathrm{C}$. Baurain, Suétone et l'inscription d'Hippo$n e$, in $L E C, 44,1976$, p. 124-126.

Bloch $1953=\mathrm{H}$. Bloch, Ostia. Iscrizioni rinvenute tra il 1930 e il 1939, in NSA, 7, 1953, p. 239-306.

Bloch $1958=\mathrm{H}$. Bloch, C. Cartilius Poplicola, in M. Floriani Squarciapino (a cura di), Le necropoli, 1. Le tombe di età repubblicana e augustea, Roma, 1958 (Scavi di Ostia, 3), p. 209-219.

Barbieri 1958 = G. Barbieri, Le iscrizioni delle necropoli, in M. Floriani Squarciapino (a cura di), Le necropoli, 1. Le tombe di età repubblicana e augustea, Roma, 1958 (Scavi di Ostia, 3), p. 131-165.

Bocherens - Zevi 2007 = C. Bocherens, F. Zevi, La «schola du Trajan» et la domus du consul Caius Fabius Agrippinus à Ostie, in ArchClass, 58, 2007, p. 257-271.

Bruun $2003=\mathrm{Ch}$. Bruun, The Antonine Plague in Rome and Ostia, in JRA, 16, 2003, p. 426-434.

Bruun $2008=$ Chr. Bruun, La familia publica a Ostia antica, in M.L. Caldelli, G.L Gregori, S. Orlandi (a cura di), Epigrafia 2006. Atti della XIV Rencontre sur l'épigraphie in onore di Silvio Panciera con altri contributi di colleghi, allievi e collaboratori (Tituli, 9), II, Roma, 2008, p. 537-556.

Caldelli 2008 = M. L. Caldelli, L'attività dei decurioni ad Ostia: funzioni e spazi, in M. Cébeillac-Gervasoni, Cl. Berrendonner, L. Lamoine (a cura di), Le quotidien municipal dans l'Occident romain (Actes du Colloque), Clermont Ferrand, 2008, p. 261-286.

Caldelli 2014, c.s. = M. L. Caldelli, Senatori oriundi di ostia : un aggiornamento, in Epigrafia e ordine senatorio: 30 anni dopo, Roma, 2014 , c.s.

Calza 1939 = G. Calza, Un documento del culto imperiale in una nuova iscrizione ostiense, in Epigraphica, 1, 1939, p. $151-157$.

Carcopino $1919=\mathrm{J}$. Carcopino, Virgile et les origines d'Ostie, Parigi, 1919.

CCCA III = M.J. Vermaseren (a cura di), Corpus cultus Cybelae Attidisque (CCCA), III, Leida, 1977.

Cébeillac-Gervasoni $2007=$ M. Cébeillac-Gervasoni, Apostilles à une énigme : le statut juridique des membres de la familia publica d'Ostie (C.I.L. XIV, 255), in Espaces et pouvoir dans l'antiquité: de l'Anatolie à la Gaule. Hommages à Bernard Remy, Grenoble, 2007, p. 159-168.
Cébeillac-Gervasoni $2010=$ M. Cébeillac-Gervasoni, in M. Cébeillac-Gervasoni, M. L. Caldelli, F. Zevi, Epigrafia latina. Ostia: cento iscrizioni in contesto, Roma, 2010.

Cébeillac-Gervasoni - Zevi $2010=$ M. CébeillacGervasoni, F. Zevi, Le tribù di Ostia, in Le tribù romane. Atti della XVI Rencontre sur l'épigraphie, Bari, 2010, p. 161-169.

Coarelli $1994=$ F. Coarelli, Saturnino, Ostia e l'annona, in Le ravitaillement en blé de Rome et des centres urbains des débuts de la République jusqu'au Haut-Empire, Actes du Colloque International organisé par le Centre J. Bérard et l'URA 994 du CNRS, Napoli, 14-16 febbr. 1991, NapoliRoma, 1994, p. 35-46.

D'Arms 1976 = J. H. D'Arms, Notes on Municipal Notables of Imperial Ostia, in AJPh, 97, 1976, p. 387-411.

D'Arms $2000=$ J. H. D'Arms, P. Lucilius Gamala's Feasts for the Ostians and their Roman Models, in JRA, 13, 2000, p. 191-200.

Demougin 1992 = S. Demougin, La promotion dans l'ordre équestre: le cas des marginaux, in La mobilité sociale dans le monde romain, Strasburgo, 1992, p. 107-121.

Demougin $1994=\mathrm{S}$. Demougin, À propos des élites locales en Italie, in L'Italie d'Auguste à Dioclétien. Actes du colloque international, Rome 25 - 28 mars 1992, Roma, 1994, p. 353-376.

Eck $1999=$ W. Eck, Ordo equitum romanorum, ordo libertorum. Freigelassene und ihre Nachkommen im römischen Ritterstand, in L'ordre équestre: histoire d'une aristocratie (II siècle av. J.-C. - III siècle ap. J.-C.), Roma, 1999, p. 5-29.

Gallina Zevi - Humphrey 2004 = A. Gallina Zevi, J. H. Humphrey (a cura di), Ostia, Cicero, Gamala, Feasts O the Economy. Papers in memory of John H. D'Arms, Ann Arbor, 2004 (JRA Suppl. 57).

Lanciani $1886=\mathrm{R}$. Lanciani, IX. Ostia, in NSA, 1886, p. $82-83$.

Gregori $2007=$ G.L. Gregori, Loca sepulturae publice data e funera publica nel Lazio d'età romana: qualche considerazione sulla documentazione epigrafica, in Seia, 14, 2007, p. 1067-1079.

Grosso 1959 = F. Grosso, L'epigrafe di Ippona e la vita di Svetonio, in RAL, ser. VIII, 14, 1959, p. 283-296.

Grosso 1964 = F. Grosso, La lotta politica al tempo di Commodo, Torino, 1964.

van Haeperen 2006 = F. van Haeperen, Interventions de Rome dans les cultes et sanctuaires de son port, Ostie, in Sanctuaires, pratiques cultuelles et territoires civiques dans l'Occident romain, Bruxelles, 2006, p. 31-50. 
Hekster 2002 = O. Hekster, Commodus. An Emperor at the Crossroads, Amsterdam, 2002.

IGI. Porto = G. Sacco (a cura di), Iscrizioni greche d'Italia . Porto, Roma, 1984.

I.It, XIII, I = A. Degrassi (a cura di), Inscriptiones Italiae, XIII, I. Fasti et Elogia. Fasti consulares et triumphales, Roma, 1947.

$I P O=\mathrm{H}$. Thylander (a cura di), Inscriptions du Port d'Ostie, I-II, Lund, 1951-52 (Skrifter utgivna av Svenska institutet i Rom, 4:1-2).

Licordari 1984 = A. Licordari, In margine ai Fasti Ostienses, in ArchClass, 36, 1984, p. 347-352.

Lo Cascio 2002 = E. Lo Cascio, Ancora sugli «Ostia's Services to Rome": collegi e corporazioni annonarie ad Ostia, in MEFRA, 114, 1, 2002, p. 87-110.

Lo Cascio $2004=$ E. Lo Cascio, Considerazioni sulla datazione di CIL XIV 375 e sulla cronologia di Gamala senior, in A. Gallina Zevi, J. H. Humphrey (a cura di), Ostia, Cicero, Gamala, Feasts $\theta$ the Economy. Papers in memory of John H. D'Arms, Ann Arbor, 2004 (JRA Suppl., 57), p. 83-88.

Lo Cascio 2012 = E. Lo Cascio (a cura di), L'impatto della "peste antonina", Bari, 2012.

López Barja de Quiroga 1995 = P. López Barja de Quiroga, Freedmen Social Mobility in Roman Italy, in Historia, 44, 3, 1995, p. 326-348.

Mar $1996=$ R. Mar, Santuarios e inversion immobiliaria en la urbanística ostiense del siglo II, in A. Gallina Zevi, A. Claridge (a cura di), "Roman Ostia» revisited. Archaeological and Historical Papers in Memory of Russell Meiggs, Roma, 1996, p. 148-164.

Marec - Pflaum 1952 = E. Marec, H.-G. Pflaum, Nouvelle inscription sur la carrière de Suétone, l'historien, in CRAI, 1952, p. 76-85.

Marinucci 1988 = A. Marinucci, Ostia. Iscrizioni municipali inedite, in $M G R, 13,1988$, p. 181-216.

Marinucci 1992 = A. Marinucci, Iscrizioni, in P. Cicerchia, A. Marinucci (a cura di), Le terme del Foro o di Gavio Massimo, Roma, 1992 (Scavi di Ostia, 11), p. 163-228.

Marinucci 2012 = A. Marinucci, Disiecta membra. Iscrizioni latine da Ostia e Porto 1981-2009, Ostia, 2012.

Meiggs $1973^{2}=$ R. Meiggs, Roman Ostia, Oxford, 19732.

Mennella 1995 = G. Mennella, I Fasti del collegium Volkani di Ostia e un nuovo frammento da Marina di Camerota, in Tra Lazio e Campania. Ricerche di storia e di topografia antica, Napoli, 1995, p. 95-101.

Nuzzo 1996 = D. Nuzzo, Impiego e reimpiego di materiale epigrafico nella basilica cristiana di Pianabella (Ostia), in VetChr, 33, 1996, p. 85-114.

Nuzzo 1999 = D. Nuzzo, Le iscrizioni, in L. Paroli et al. (a cura di), La basilica cristiana di Pianabella, Roma, 1999 (Scavi di Ostia, 12), p. 33-202.

Panciera $2004=\mathrm{S}$. Panciera, Considerazioni intorno a CIL $X I V$ 375, in A. Gallina Zevi, J. H. Humphrey (a cura di), Ostia, Cicero, Gamala, Feasts $\theta$ the Economy. Papers in memory of John H. D'Arms, Ann Arbor, 2004 (JRA Suppl., 57), p. 69-74 [ripresa in Epigrafi, epigrafia, epigrafisti. Scritti vari editi e inediti (1956 - 2005) con note complementari e indici, Roma, 2006, p. 103-110].

Pavis d'Escurac $1974=\mathrm{H}$. Pavis d'Escurac, Réflexions sur la classis Africana Commodiana, in Mélanges d'historie ancienne offerts à William Seston, Parigi, 1974, p. 397-408.
Pavis d'Escurac $1976=\mathrm{H}$. Pavis d'Escurac, La préfecture de l'annone, service administratif impérial d'Auguste à Constantin, Roma, 1976 (BEFAR, 226).

Pellegrino $1986=$ A. Pellegrino, Il culto di Vulcano ad Ostia Nuove testimonianze, in $M G R, 10,1986$, p. 289-301.

Pellegrino 1988 = A. Pellegrino, Note sul culto di Serapide ad Ostia, in MGR, 13, 1988, p. 225-242.

Pensabene 2002 = P. Pensabene, Committenza edilizia $a$ Ostia tra la fine del I e i primi decenni del III secolo : lo studio dei marmi e della decorazione architettonica come strumento d'indagine, in MEFRA, 114, 1, 2002, p. 181-324.

$P I R^{2}=$ Prosopographia Imperii Romani. Saec. I, II, III, Berlino, 1933-.

Pohl 1978a = I. Pohl, Piazzale delle Corporazioni ad Ostia: tentativo di ricostruzione del portico claudio e la sua decorazione, in MEFRA, 90, 1, 1978, p. 331-355.

Pohl 1978b = I. Pohl, in F. Zevi, I. Pohl, M. Carta (a cura di), Ostia. La Taberna dell'invidioso. Piazzale delle corporazioni, portico ovest. Saggi sotto i mosaici, in NSA, 32, 1978 (Suppl.), p. 165-443.

RICIS $=$ L. Bricault (a cura di), Recueil des inscriptions concernant les cultes isiaques (RICIS), I-III, Parigi, 2005.

Rickman 1971 = G. Rickman, Roman Granaries and Store Buildings, Cambridge, 1971.

Rieger 2004 = A.-K. Rieger, Heiligtümer in Ostia, Monaco, 2004.

Royden $1988=$ H. L. Royden, The Magistrates of the Roman Professional Collegia in Italy from the First to the Third Century A.D., Pisa, 1988.

Salomies 1996 = O. Salomies, Senatori oriundi del Lazio, in H. Solin (a cura di), Studi storico-epigrafici sul Lazio antico, Roma, 1996, p. 23-127 (Acta Inst. Rom. Finl., 15).

Salomies 2002= O. Salomies, People in Ostia : some Onomastic Observations and Comparisons with Rome, in Ch. Bruun, A. Gallina Zevi (a cura di), Ostia e Portus nelle loro relazioni con Roma, Roma, 2002 (ActaInstRomFin, 27), p. 135-159.

Salomies $2003=$ O. Salomies, A study of CIL XIV 375, an interesting inscription from Ostia, in Arctos, 37, 2003, p. 133-157.

Salomies $2010=$ O. Salomies, Aedilicius, consularis, duumviralis and Similar Titles in Latin Inscriptions, in Arctos, 44, 2010, p. 205-229.

Scheid 1999 = J. Scheid, Les sacerdoces publics équestres, in L'ordre équestre: histoire d'une aristocratie (II' siècle av. J.-C. - III siècle ap. J.-C.), Roma, 1999, p. 79-96, 114 $147,185-189$.

Sijpesteijn $1996=$ P. J. Sijpesteijn, Lateinische Grabinschriften $I V$, in ZPE, 111,1996, p. 283-288.

Steuernagel $2004=$ D. Steuernagel, Kult und Alltag in römischen Hafenstädten. Soziale Prozesse in archäologischer Perspektive, Monaco, 2004.

Storchi Marino 2012 = A. Storchi Marino, Una rilettura delle fonti storico-letterarie sulla peste di età antonina, in E. Lo Cascio (a cura di), L'impatto della "peste antonina", Bari, 2012, p. 31-61.

Sudi-Guiral 2007 = Fr. Sudi-Guiral, La familia publica d'Ostie, in MEFRA, 119, 2007, p. 421-426.

Vidman 1957 = L. Vidman, Fasti Ostienses. Edendos illustrandos restituendos curavit, Praga, 1957. 
Vidman 1969 = L. Vidman, Sylloge inscriptionum religionis Isiacae et Serapiacae, Berlino, 1969.

Vidman 19822 = L. Vidman, Fasti Ostienses. Edendos illustrandos restituendos curavit, Praga, 1982².

Wardle 2002 = D. Wardle, Suetonius as ab epistulis: an African Connection, in Historia, 51, 4, 2002, p. 465-470.

Whittaker $1964=$ C.R. Whittaker, The Revolt of Papirius Dionysius A.D. 190, in Historia, 13, 1964, p. 348-369.

Zevi 1970 = F. Zevi, Nuovi documenti epigrafici sugli Egrili ostiensi, in MEFRA, 82, 1970, p. 289-308.

Zevi 1973 = F. Zevi, P. Lucilio Gamala senior e i quattro tempietti di Ostia, in MEFRA, 85, 1973, p. 555-581.

Zevi 2004 = F. Zevi, Ancora su T. Statilius Taurianus e il Serapeo di Ostia, in Epigraphica, 66, 2004, p. 95-108.

Zevi 2004 = F. Zevi, P. Lucilio Gamala senior: un riepilogo trent'anni dopo, in A. Gallina Zevi, J. H. Humphrey (a cura di), Ostia, Cicero, Gamala, Feasts $\theta$ the Economy. Papers in memory of John H. D'Arms, Ann Arbor, 2004, (JRA Suppl., 57), p. 47-67.

Zevi 2008 = F. Zevi, I collegi di Ostia e le loro sedi associative tra Antonini e Severi, in C. Berrendonner, M. CébeillacGervasoni, L. Lamoine (a cura di), Le quotidien municipal dans l'Occident romain, Clermont-Ferrand, 2008, p. 477-505.

Zevi $2009=$ F. Zevi, Catone e $i$ cavalieri grassi. Il culto di Vulcano ad Ostia. Una proposta di lettura storica, in MEFRA, 121, 2009, p. 503-513.

Zevi $2010=$ F. Zevi, in M. Cébeillac-Gervasoni, M. L. Caldelli, F. Zevi, Epigrafia latina. Ostia: cento iscrizioni in contesto, Roma, 2010.

Zevi 2012 = F. Zevi, Culti ed edifici templari di Ostia repubblicana, in Ostraka (vol. spec.), 2012, p. 537-563. 
